\title{
GAMMA DISTRIBUTIONS FOR STATIONARY POISSON FLAT PROCESSES
}

\author{
VOLKER BAUMSTARK * AND \\ GÜNTER LAST, ${ }^{* * *}$ Universität Karlsruhe
}

\begin{abstract}
We consider a stationary Poisson process $X$ of $k$-flats in $\mathbb{R}^{d}$ with intensity measure $\Theta$ and a measurable set $S$ of $k$-flats depending on $F_{1}, \ldots, F_{n} \in X, x \in \mathbb{R}^{d}$, and $X$ in a specific equivariant way. If $\left(F_{1}, \ldots, F_{n}, x\right)$ is properly sampled (in a 'typical way') then $\Theta(S)$ has a gamma distribution. This result generalizes and unifies earlier work by Miles (1971), Møller and Zuyev (1996), and Zuyev (1999). As a new example, we will show that the volume of the fundamental region of a typical $j$-face of a stationary PoissonVoronoi tessellation is conditionally gamma distributed. This is true in the area-biased and the area-debiased cases. In the first case the shape parameter is not integer valued. As another new example, we will show that the generalized integral-geometric contents of the (area-biased and area-debiased) typical $j$-face of a Poisson hyperplane tessellation are conditionally gamma distributed. In the isotropic case the contents boil down to the mean breadth of the face.
\end{abstract}

Keywords: Stochastic geometry; gamma distribution; $k$-flat; Poisson process; Palm distribution; stopping set; Voronoi tessellation; Poisson hyperplane tessellation; typical face

2000 Mathematics Subject Classification: Primary 60D05; 60G55

\section{Introduction}

Since the seminal work in [8] and [12], it is known that the (generalized) integral-geometric contents of several closed sets constructed on stationary Poisson processes of flats are (conditionally) gamma distributed. It is also known (see [17]) that the measure of certain stopping sets defined on Poisson processes is conditionally gamma distributed. These are some of the rare cases in stochastic geometry where the distribution of nontrivial geometric functionals of Poisson processes is explicitly known. Further results of this type can, e.g. be found in [1], [2], [3], [6], and [13], with some corrections to the work in [8] and [12] given in [2]. The aim of this paper is to generalize these results using a unified framework combining stopping sets with Palm distributions. Several examples will illustrate the theory.

We consider here a stationary Poisson process $X$ of $k$-flats ( $k$-dimensional affine subspaces) in $\mathbb{R}^{d}$, where $d \geq 1$ and $k \in\{0, \ldots, d-1\}$. This is a Poisson process on the space of all $k$-flats, whose distribution is invariant under translation of the flats. Its distribution is determined by the intensity $\gamma$ (see (2.2), below), and the directional distribution $\mathbb{Q}$, a probability measure $\mathbb{Q}$ on the space of all $k$-dimensional linear subspaces of $\mathbb{R}^{d}$. In fact, the intensity measure of $X$ equals $\gamma \Theta$, where $\Theta$ is the intensity measure of a stationary Poisson process of $k$-flats with intensity 1 (see (2.3), below). Note that $X$ is isotropic, if and only if $\mathbb{Q}$ is the uniform distribution. If $k=0$

Received 30 August 2007; revision received 19 June 2009.

* Postal address: Institut für Stochastik, Universität Karlsruhe (TH), 76128 Karlsruhe, Germany.

** Email address: last@math.uni-karlsruhe.de 
then $X$ is just a stationary Poisson process on $\mathbb{R}^{d}$. We refer the reader to [14] and [15] for these and other fundamental facts from stochastic geometry.

To describe and motivate the main results of this paper, we first discuss a special case. Let $\mathcal{E}^{k}$ denote the space of all $k$-flats in $\mathbb{R}^{d}$ equipped with the usual topology; see [14]. Fix $n \in \mathbb{N}$. Let $S\left(F_{1}, \ldots, F_{n}, x\right)$ be a Borel subset of $\varepsilon^{k}$ depending on $F_{1}, \ldots, F_{n} \in \mathcal{E}^{k}$, and let $x \in \mathbb{R}^{d}$ in a measurable way. Assume that $S$ is equivariant in the sense that a joint scaling or translation of all arguments leads to the same scaling or translation of the elements of $S$. Furthermore, let $R\left(F_{1}, \ldots, F_{n}, x, X\right)$ be a nonnegative measurable mapping, invariant under joint scaling or translation of all arguments. In many examples $R$ is just an indicator function. Fix $m \in \mathbb{N}_{0}$, let $\eta \subset \mathcal{E}^{k}$ be locally finite, and define $R_{m}\left(F_{1}, \ldots, F_{n}, x, \eta\right):=R\left(F_{1}, \ldots, F_{n}, x, \eta\right)$ if $\operatorname{card}(\eta \cap$ $\left.S\left(F_{1}, \ldots, F_{n}, x\right)\right)=m$ and $R_{m}\left(F_{1}, \ldots, F_{n}, x, \eta\right):=0$ otherwise. Let $\mu\left(F_{1}, \ldots, F_{n}, \cdot\right)$ be a measure on $\mathbb{R}^{d}$ with the equivariance property (3.2), below. We assume that $\mu$ is a kernel from $\left(\varepsilon^{k}\right)^{n}$ to $\mathbb{R}^{d}$, that is, $\mu\left(F_{1}, \ldots, F_{n}, \cdot\right)$ is measurable in the first $n$ arguments and $\sigma$-finite in the last argument. Let $B \subset \mathbb{R}^{d}$ and $C \subset[0, \infty)$ be Borel sets, and define $M(B \times C)$ by summing

$$
\begin{aligned}
& \int \mathbf{1}\left\{x \in B, \Theta\left(S\left(F_{1}, \ldots, F_{n}, x\right)\right) \in C\right\} R_{m}\left(F_{1}, \ldots, F_{n}, x, X \backslash\left\{F_{1}, \ldots, F_{n}\right\}\right) \\
& \quad \times \mu\left(F_{1}, \ldots, F_{n}, \mathrm{~d} x\right)
\end{aligned}
$$

over all pairwise different $F_{1}, \ldots, F_{n} \in X$. This gives a stationary random marked measure $M$ on $\mathbb{R}^{d}$ with mark space $[0, \infty)$. It is easy to see (and derived in Section 3 ) that the intensity $\gamma_{M}:=\mathrm{E} M\left([0,1]^{d} \times[0, \infty)\right)$ of $M$ is given by

$$
\begin{aligned}
\gamma_{M}=\gamma^{n} \iint & \mathbf{1}\left\{x \in[0,1]^{d}\right\} \mathrm{E}\left[R_{m}\left(F_{1}, \ldots, F_{n}, x, X\right)\right] \mu\left(F_{1}, \ldots, F_{n}, \mathrm{~d} x\right) \\
& \times \Theta^{n}\left(\mathrm{~d}\left(F_{1}, \ldots, F_{n}\right)\right) .
\end{aligned}
$$

If this intensity is positive and finite then $\mathrm{E} M(B \times C)=\gamma_{M} \mathscr{H}^{d}(B) \mathbb{V}(C)$ for any Borel sets $B \subset \mathbb{R}^{d}$ and $C \subset[0, \infty)$, where $\mathscr{H}^{d}$ is the Lebesgue measure and $\mathbb{V}$ is a distribution on $[0, \infty)$, the mark distribution of $M$.

The following theorem generalizes both Miles' complementary theorem in [8] (see also [12]), and the results on subprocesses in [12]. We will discuss these cases in Section 4. Later in the paper we will extend the theorem to situations where the set $S$ may also depend on the flat process $X$; see Theorem 3.1, below. The gamma distribution with shape parameter $\alpha>0$ and scale parameter $\gamma$ is denoted by $\Gamma(\alpha, \gamma)$.

Theorem 1.1. Let $M$ be the stationary random marked measure defined above, and assume that its intensity is positive and finite. Let $j \in\{0, \ldots, d\}$ satisfy $m+n-(d-j) /(d-k)>0$, and assume that the kernel $\mu$ satisfies the scaling relation

$$
\mu\left(F_{1}, \ldots, F_{n}, c^{-1} B\right)=c^{-j} \mu\left(c F_{1}, \ldots, c F_{n}, B\right), \quad F_{1}, \ldots, F_{n} \in \mathcal{E}^{k}, c>0,
$$

for any Borel set $B \subset \mathbb{R}^{d}$. Then the mark distribution of $M$ is $\Gamma(m+n-(d-j) /(d-k), \gamma)$.

To give the reader some feeling for the random measure $M$ and the assertion of Theorem 1.1, we continue with some examples.

Example 1.1. Let $k=0$, and consider the Voronoi tessellation based on the Poisson process $X$; see also Section 5. Let $j \in\{0, \ldots, d\}$. Pick a point $x$ on one of the $j$-faces 'at random' and consider the ball centered at $x$, having $d-j+1$ points of $X$ (the neighbors of the $j$-face) on its 
boundary but no point of $X$ in its interior. Then the volume of this ball has a $\Gamma(d-j+j / d, \gamma)$ distribution. For $j=0$, this result is due to Miles [9], while the $j=d$ case is trivial. The other cases can be found in [1] and [13].

To derive this result from Theorem 1.1, we consider points $x_{1}, \ldots, x_{d-j+1} \in X$ in general position. Let $Z_{j}\left(x_{1}, \ldots, x_{d-j+1}\right)$ be the set of all points $x \in \mathbb{R}^{d}$ having the same distance from all the points $x_{1}, \ldots, x_{d-j+1} \in X$. For such $x$, we let $S\left(x_{1}, \ldots, x_{d-j+1}, x\right)$ be the closed ball $B\left(x,\left|x_{1}-x\right|\right)$ centered at $x$ and having the points $x_{1}, \ldots, x_{d-j+1}$ on its boundary. A point $x \in Z_{j}\left(x_{1}, \ldots, x_{d-j+1}\right)$ is in a $j$-face of the tessellation if $S\left(x_{1}, \ldots, x_{d-j+1}, x\right)$ does not contain any further points of $X$. Define $M(B \times C)$ by summing

$$
\begin{aligned}
\int_{B} \mathbf{1}\left\{\mathscr{H}^{d}\left(S\left(x_{1}, \ldots, x_{d-j+1}, x\right)\right) \in C\right\} \\
\quad \times \mathbf{1}\left\{\left(X \backslash\left\{x_{1}, \ldots, x_{d-j+1}\right\}\right) \cap S\left(x_{1}, \ldots, x_{d-j+1}, x\right)=\varnothing\right\} \mu\left(x_{1}, \ldots, x_{d-j+1}, \mathrm{~d} x\right)
\end{aligned}
$$

over all $x_{1}, \ldots, x_{d-j+1} \in X$ in general position, where

$$
\mu\left(x_{1}, \ldots, x_{d-j+1}, \mathrm{~d} x\right):=\mathbf{1}\left\{x \in Z_{j}\left(x_{1}, \ldots, x_{d-j+1}\right)\right\} \mathscr{H}^{j}(\mathrm{~d} x)
$$

and $\mathscr{H}^{j}$ is a $j$-dimensional Hausdorff measure on $\mathbb{R}^{d}$. Then Theorem 1.1 applies with $n:=$ $d-j+1$ and $m:=0$. Hence, $\mathbb{V}$ indeed equals $\Gamma(d-j+j / d, \gamma)$.

Example 1.2. As in Example 1.1, we let $k=0$ and consider the Voronoi tessellation based on $X$. We let $j \in\{0, \ldots, d\}$ and pick one of the $j$-faces 'at random'. This face then has $d-j+1$ neighbors $x_{1}, \ldots, x_{d-j+1} \in X$. Let $z\left(x_{1}, \ldots, x_{d-j+1}\right)$ be the center of the $(d-$ $j$ )-dimensional ball having the above neighbors on its boundary. Then the volume of the ( $d$-dimensional) ball $S\left(x_{1}, \ldots, x_{d-j+1}\right)$ centered at $z\left(x_{1}, \ldots, x_{d-j+1}\right)$ and having $x_{1}$ on its boundary is conditionally $\Gamma(d-j+m, \gamma)$-distributed given that $X \backslash\left\{x_{1}, \ldots, x_{d-j+1}\right\}$ has $m \in \mathbb{N}_{0}$ points in $S\left(x_{1}, \ldots, x_{d-j+1}\right)$. This fact seems to be new. Note that $z\left(x_{1}, \ldots, x_{d-j+1}\right)$ need not lie on the picked $j$-face. This result can be formally obtained as follows. Consider points $x_{1}, \ldots, x_{d-j+1} \in X$ in general position and define $z\left(x_{1}, \ldots, x_{d-j+1}\right)$ as above. Let $\eta \subset \mathcal{E}^{k}$ be locally finite, set $R\left(x_{1}, \ldots, x_{d-j+1}, \eta\right):=1$ if

$$
L_{j}\left(x_{1}, \ldots, x_{d-j+1}, \eta\right):=\left\{x \in Z_{j}\left(x_{1}, \ldots, x_{d-j+1}\right): \eta \cap B^{0}\left(x,\left|x-x_{1}\right|\right)=\varnothing\right\}
$$

has nonempty relative interior, and set $R\left(x_{1}, \ldots, x_{d-j+1}, \eta\right):=0$ otherwise. (In the first case $L_{j}\left(x_{1}, \ldots, x_{d-j+1}, X \backslash\left\{x_{1}, \ldots, x_{d-j+1}\right\}\right)$ is a $j$-face.) Here $Z_{j}\left(x_{1}, \ldots, x_{d-j+1}\right)$ is (again) the set of all points $x \in \mathbb{R}^{d}$ having the same distance from all the points $x_{1}, \ldots, x_{d-j+1} \in X$ and $B^{0}\left(x,\left|x-x_{1}\right|\right)$ is the interior of the ball $B\left(x,\left|x-x_{1}\right|\right)$. Let $S\left(x_{1}, \ldots, x_{d-j+1}\right)$ be the ball $B\left(z\left(x_{1}, \ldots, x_{d-j+1}\right),\left|x_{1}-z\left(x_{1}, \ldots, x_{d-j+1}\right)\right|\right)$. Define $M(B \times C)$ by summing

$$
\mathbf{1}\left\{\mathscr{H}^{d}\left(S\left(x_{1}, \ldots, x_{d-j+1}\right)\right) \in C\right\} R_{m}\left(x_{1}, \ldots, x_{d-j+1}, x, X \backslash\left\{x_{1}, \ldots, x_{d-j+1}\right\}\right)
$$

over all $x_{1}, \ldots, x_{d-j+1} \in X$ in general position and having $z\left(x_{1}, \ldots, x_{d-j+1}\right) \in B$. Let $\mu\left(x_{1}, \ldots, x_{d-j+1}, \cdot\right)$ be the Dirac measure at $z\left(x_{1}, \ldots, x_{d-j+1}\right)$. Since $z(\cdot)$ is equivariant under scaling, (1.2) holds with $j=0$. Theorem 1.1 with $n:=d-j+1$ shows that $\mathbb{V}$ is a gamma distribution with shape parameter $d-j+1+m-1=d-j+m$.

Example 1.3. Let $k=d-1$, and assume that $X$ is nondegenerate, i.e. not all hyperplanes in $X$ are parallel to a fixed line. Then $X$ induces a tessellation of $\mathbb{R}^{d}$ (a Poisson hyperplane tessellation); see Theorem 10.3.2 of [14]. Pick one of the cells at random and consider the 
(maximal) inradius of this cell. This radius is exponentially distributed. This is Theorem 10.4.11 of [14], where the result is attributed to Miles. The result is also treated as Example (vi) in [12] in the setting of Miles' complementary theorem. (As pointed out in [14], this requires additional properties of the directional distribution $\mathbb{Q}$.)

We now develop a version of this result, where only cells with unique inball are being selected. For $F_{1}, \ldots, F_{d+1} \in \mathcal{E}^{d-1}$, let $\S_{d}\left(F_{1}, \ldots, F_{d+1}\right)$ denote the system of the closures of the connected components of the complement of $F_{1} \cup \cdots \cup F_{d}$. Define $R\left(F_{1}, \ldots, F_{d+1}\right):=1$ if there is exactly one cell in $\delta_{d}\left(F_{1}, \ldots, F_{d+1}\right)$ that has a unique inball $\tilde{S}\left(F_{1}, \ldots, F_{d+1}\right)$. In this case we let $z\left(F_{1}, \ldots, F_{d+1}\right)$ denote the center of this inball and $r\left(F_{1}, \ldots, F_{d+1}\right)$ denote its radius. In all other cases we set $R\left(F_{1}, \ldots, F_{d+1}\right):=0$. The random measure $M$ is obtained by summing

$$
\mathbf{1}\left\{z\left(F_{1}, \ldots, F_{d+1}\right) \in B, 2 r\left(F_{1}, \ldots, F_{d+1}\right) \in C\right\} R_{0}\left(F_{1}, \ldots, F_{d+1}, x, X \backslash\left\{F_{1}, \ldots, F_{d+1}\right\}\right)
$$

over all pairwise different $F_{1}, \ldots, F_{d+1} \in X$. It follows from Theorem 10.3.3 of [14] that the intensity $\gamma_{M}$ is finite. Let $S\left(F_{1}, \ldots, F_{d+1}\right):=\left\{F \in \mathcal{E}^{d-1}: F \cap \tilde{S}\left(F_{1}, \ldots, F_{d+1}\right) \neq \varnothing\right\}$. Then $\Theta\left(S\left(F_{1}, \ldots, F_{d+1}\right)\right)=2 r\left(F_{1}, \ldots, F_{d+1}\right)$. Theorem 1.1 with $n:=d+1, m:=0$, and $j:=0$ shows that $\mathbb{V}$ is a gamma distribution with shape parameter $d+1-d=1$.

Example 1.4. As in Example 1.3, we consider a nondegenerate Poisson hyperplane tessellation. Pick a point $x$ on the (one-dimensional) edges 'at random'. Then $x$ is in the relative interior of an edge $L_{1}$. Define $\Theta^{*}\left(L_{1}\right)$ as the $\Theta$-measure of all $F \in \mathcal{E}^{d-1}$ having $F \cap L_{1} \neq \varnothing$; see also (2.4), below. Then $\Theta^{*}\left(L_{1}\right)$ has a $\Gamma(2, \gamma)$-distribution. In the isotropic case, $\Theta^{*}\left(L_{1}\right)$ is proportional to the length of $L_{1}$. By a Slivnyak-type argument and area-biased sampling of Poisson intervals, the result is then easy to guess. In the general case the assertion is less obvious.

To derive the result, we let $F_{1}, \ldots, F_{d+1} \in \mathcal{E}^{d-1}$. If $F_{1} \cap \cdots \cap F_{d-1}$ is of dimension 1 , we let $\mu\left(F_{1}, \ldots, F_{d+1}, \cdot\right)$ be the one-dimensional Hausdorff measure supported by this intersection. Take $x \in F_{1} \cap \cdots \cap F_{d-1}$, and define

$$
L_{1}\left(F_{1}, \ldots, F_{d+1}, x\right):=F_{1} \cap \cdots \cap F_{d-1} \cap F_{d}(x) \cap F_{d+1}(x),
$$

where $F_{i}(x)(i=d, d+1)$ is the half-space bounded by $F_{i}$ and containing $x$. (For $x \in F_{i}$, we let $F_{i}(x):=\mathbb{R}^{d}$.) Let $R\left(F_{1}, \ldots, F_{d+1}, x\right):=1$ if the set (1.3) is a bounded segment, and set $R\left(F_{1}, \ldots, F_{d+1}, x\right):=0$ otherwise. Define $M(B \times C)$ by summing

$$
\begin{aligned}
& \int \mathbf{1}\left\{x \in B, \Theta^{*}\left(L_{1}\left(F_{1}, \ldots, F_{d+1}, x\right)\right) \in C\right\} R\left(F_{1}, \ldots, F_{d+1}, x\right) \\
& \quad \times \mathbf{1}\left\{\left(X \backslash\left\{F_{1}, \ldots, F_{d+1}\right\}\right) \cap L_{1}\left(F_{1}, \ldots, F_{d+1}, x\right)=\varnothing\right\} \mu\left(F_{1}, \ldots, F_{d+1}, \mathrm{~d} x\right)
\end{aligned}
$$

over all pairwise different $F_{1}, \ldots, F_{d+1} \in X$. Theorem 10.3 .3 of [14] implies that $M$ has a finite intensity. Then Theorem 1.1 applies with $n:=d+1, m:=0, j:=1$, and

$$
S\left(F_{1}, \ldots, F_{d+1}, x\right):=\left\{F \in \mathcal{E}^{d-1}: F \cap L_{1}\left(F_{1}, \ldots, F_{d+1}, x\right) \neq \varnothing\right\} .
$$

Hence, $\mathbb{V}$ is a gamma distribution with shape parameter 2 .

Example 1.5. As in Examples 1.3 and 1.4, we consider a nondegenerate Poisson hyperplane tessellation. Pick an edge $C_{1}$ 'at random'. Then $\Theta^{*}\left(C_{1}\right)$ has an exponential distribution. This can be proved as in Example 1.4. 
For the reader's convenience, we sketch a proof of Theorem 1.1 in case the function $R$ does not depend on $X$. By definition, $\mathbb{V}(C)=\gamma_{M}^{-1} \mathrm{E} M\left([0,1]^{d} \times C\right)$. Using an iterated version of Mecke's Satz 3.1 in [7], we obtain

$$
\mathbb{V}(C)=\frac{\gamma^{n}}{\gamma_{M} m !} \int \mathbf{1}\left\{x \in[0,1]^{d}, t \in C\right\} \exp [-\gamma t] v_{m}(\mathrm{~d}(x, t)),
$$

where, for any $b \in \mathbb{R}$, the measure $v_{b}$ on $\mathbb{R}^{d} \times(0, \infty)$ is defined by

$$
\begin{aligned}
v_{b}(B \times C):=\iint & \mathbf{1}\left\{x \in B, \Theta\left(S\left(F_{1}, \ldots, F_{n}, x\right)\right) \in C\right\} \Theta\left(S\left(F_{1}, \ldots, F_{n}, x\right)\right)^{b} \\
& \times R\left(F_{1}, \ldots, F_{n}, x\right) \mu\left(F_{1}, \ldots, F_{n}, \mathrm{~d} x\right) \Theta^{n}\left(\mathrm{~d}\left(F_{1}, \ldots, F_{n}\right)\right) .
\end{aligned}
$$

This usage of Mecke's formula (also called the Mecke-Slivnyak formula) is by now very standard in stochastic geometry. Fix $b \in \mathbb{R}$ for a moment. From equivariance of $S$ and $R$ under translations we find that $v_{b}$ is translation invariant in the first component. Hence,

$$
v_{b}(B \times C)=v_{b}\left([0,1]^{d} \times C\right) \mathcal{H}^{d}(B) .
$$

(If $C$ is bounded away from 0 and $\infty$, we can use the fact that the integral in (1.1) is assumed finite.) Now fix a Borel set $C \subset(0, \infty)$ and let $a>0$. By a straightforward calculation,

$$
v_{b}\left([0,1]^{d} \times a C\right)=a^{n} a^{b} a^{j /(d-k)} v_{b}\left(a^{-1 /(d-k)}[0,1]^{d} \times C\right),
$$

where the first two factors come from the form of the measure $\Theta,(2.3)$, below, while the third comes from the scaling relation (1.2). Choosing $b:=1-n+(d-j) /(d-k)$ we find from (1.5) that $v_{b}\left([0,1]^{d} \times a C\right)=a v_{b}\left([0,1]^{d} \times C\right)$. Hence, $v_{b}\left([0,1]^{d} \times C\right)=c \mathscr{H}^{1}(C)$ for some $c>0$. Inserting this, (1.5), and the obvious equation

$$
v_{m}(B \times C)=\int \mathbf{1}\{x \in B, t \in C\} t^{m-b} v_{b}(\mathrm{~d}(x, t))
$$

into (1.4) we find that $\mathbb{V}$ is proportional to

$$
\int \mathbf{1}\left\{x \in[0,1]^{d}, t \in \cdot\right\} t^{m-b} \exp [-\gamma t] \nu_{b}(\mathrm{~d}(x, t))=\int \mathbf{1}\{t \in \cdot\} t^{m-b} \exp [-\gamma t] \mathrm{d} t,
$$

as claimed.

As already mentioned above, we will show that Theorem 1.1 remains valid in the more general case where the set $S$ may also depend on the flat process $X$. Our crucial assumption is that $S\left(F_{1}, \ldots, F_{n}, x, \cdot\right)$ is a stopping set for all $k$-flats $F_{1}, \ldots, F_{n}$ and all $x \in \mathbb{R}^{d}$. Moreover, the function $R\left(F_{1}, \ldots, F_{n}, x, \cdot\right)$ is assumed to be measurable with respect to the associated stopping $\sigma$-field. It is then reasonable to include the $n=0$ case by defining

$$
M(B \times C):=\int \mathbf{1}\{x \in B, \Theta(S(x, X)) \in C, \operatorname{card}(X \cap S(x, X))=m\} R(x, X) \mathrm{d} x .
$$

This way we can also cover the results of [17], noting, however, that the latter paper studies stopping sets defined on more general Poisson processes. We believe that our approach can be used to explain all gamma-type results for Poisson flat processes.

A finite intensity of the underlying random measure is a crucial assumption in Theorem 1.1 and the more general Theorem 3.1, below. Cowan [2] showed what can go wrong with Miles' 
complementary theorem without this assumption. As a resort, he proposed an approach via a limit procedure that could easily be adapted to our more general setting. We are not pursuing this here, mainly because the results seem to be rather preliminary. But there is certainly room for further research on the topic of this paper.

The structure of this paper is as follows. Section 2 contains some technical prerequisites that are required for a sound mathematical treatment of our topic. In Section 3 we present our main results (Theorem 3.1 and Theorem 3.2) and their proofs. Even in the situation where the set $S$ does not depend on $X$, Theorem 3.1 will be more general than Theorem 1.1. In Section 4 we will discuss (and slightly generalize) the classical special cases mentioned above. In Section 5 we will present new specific examples in the $k=0$ case, i.e. in the case where $X$ is a stationary Poisson process. We will consider area-biased and area-debiased versions of the typical $j$-face of the Voronoi tessellation based on $X$. The volume of the fundamental region of this face has a conditional gamma distribution given the number of $X$ points in this region. The area-debiased version for $j=d$ can be found in [10] and [12], while the area-debiased version for $j=1$ can be found in [3] (for $d=2$ ) and in [1]. The classical case, $j=0$, has been treated in [9]. All other cases seem to be new. In Section 6 we consider area-biased and area-debiased typical $j$-faces of a general Poisson hyperplane tessellation. The generalized integral-geometric contents of such a $j$-face are conditionally gamma distributed given its number of $(j-1)$-dimensional faces. In the isotropic case the contents are the mean breadth of the face. This section generalizes classical results on the typical cell of a Poisson hyperplane tessellation; see [6, Section 6.3] and [12]. Appendix A contains some (apparently new) material on stopping sets that is needed for proving our results.

\section{Preliminaries}

\subsection{Geometrical preliminaries}

We work in Euclidean $d$-space $\mathbb{R}^{d}, d \geq 1$, equipped with the Euclidean norm $|\cdot|$ and the Borel $\sigma$-field $\mathscr{B}^{d}$. The closed ball with radius $r \geq 0$ centered at $x \in \mathbb{R}^{d}$ is denoted by $B(x, r)$, while $B^{0}(x, r)$ denotes the corresponding open ball. The unit ball $B(0,1)$ centered at the origin $0 \in \mathbb{R}^{d}$ is denoted by $B^{d}$. We write $\mathscr{H}^{k}$ for the $k$-dimensional Hausdorff measure in $\mathbb{R}^{d}$, and let $\kappa_{d}:=\mathscr{H}^{d}\left(B^{d}\right)$.

The system of all closed subsets of $\mathbb{R}^{d}$ is denoted by $\mathcal{F}$. For any $K \subset \mathbb{R}^{d}$, we write

$$
\mathcal{F}_{K}:=\{F \in \mathcal{F}: F \cap K \neq \varnothing\} .
$$

We make $\mathcal{F}$ a measurable space by introducing the smallest $\sigma$-field containing $\mathcal{F}_{K}$ for all compact $K$. This is actually the Borel $\sigma$-field associated with the topology of closed convergence on $\mathcal{F}$; see [14, Chapter 12] for more detail. If $K \subset \mathbb{R}^{d}$ is compact then $\mathcal{F}_{K}$ is compact with respect to this topology. Conversely, any compact subset of $\mathcal{F} \backslash\{\varnothing\}$ is contained in $\mathcal{F}_{K}$ for some compact $K$. Let $k \in\{0, \ldots, d-1\}$. A $k$-flat is a $k$-dimensional affine subspace $F$ of $\mathbb{R}^{d}$. The space of all such flats is denoted by $\mathcal{E}^{k}$. Any $F \in \mathcal{E}^{k}$ can be uniquely written as $F=L+x$, where $L$ is an element of the space $\mathcal{L}^{k}$ of all $k$-dimensional linear subspaces of $\mathbb{R}^{d}$ and $x$ is in the orthogonal complement $L^{\perp}$ of $L$. The sets $\mathcal{E}^{k}$ and $\mathcal{L}^{k}$ are respectively locally compact and compact second countable Hausdorff spaces; see Theorems 13.2.4 and 13.2.5, and the subsequent remarks of [14]. The space of all closed subsets of $\mathcal{E}^{k}$ is denoted by $\mathcal{F}\left(\mathcal{E}^{k}\right)$. Again, this space can be equipped with the topology of closed convergence. In particular, $\mathcal{F}\left(\mathcal{E}^{k}\right)$ becomes a measurable space in its own right. Sometimes we need the special notation

$$
\mathcal{E}_{K}^{k}:=\mathcal{F}_{K} \cap \mathcal{E}^{k}=\left\{F \in \mathcal{E}^{k}: F \cap K \neq \varnothing\right\}, \quad K \in \mathcal{F} .
$$




\subsection{Stationary Poisson processes of flats}

We let $N^{k}$ denote the space of all locally finite subsets of $\mathcal{E}^{k}$. Any $\eta \in \boldsymbol{N}^{k}$ is identified with the counting measure $A \mapsto \eta(A):=\operatorname{card}(\eta \cap A)$ on $\mathcal{E}^{k}$. The $\sigma$-field $\mathcal{N}^{k}$ is the smallest $\sigma$-field on $N^{k}$ making the mappings $\eta \mapsto \eta(A)$ measurable for all Borel sets $A \subset \mathcal{E}^{k}$. A point process of $k$-flats is a point process on $\mathcal{E}^{k}$, i.e. a measurable mapping $X$ from some abstract probability space $(\Omega, \mathcal{A}, \mathrm{P})$ into $N^{k}$. It is called stationary if $X+x$ has the same distribution as $X$ for all $x \in \mathbb{R}^{d}$. Here $\eta+x:=\{F+x: F \in \eta\}$ for $\eta \in N^{k}$. Consider a stationary point process of $k$-flats and assume that its intensity

$$
\gamma:=\frac{1}{\kappa_{d-k}} \operatorname{Ecard}\left(F \in X: F \cap B^{d} \neq \varnothing\right)
$$

is finite and positive. Then the intensity measure of $X$ can be written as

$$
\mathrm{E} X(A)=\gamma \Theta(A)
$$

where

$$
\Theta(\cdot):=\int_{\mathscr{L}^{k}} \int_{F^{\perp}} 1\{F+x \in \cdot\} \mathscr{H}^{d-k}(\mathrm{~d} x) \mathbb{Q}(\mathrm{d} F)
$$

for some uniquely determined probability measure $\mathbb{Q}$ on $\mathcal{L}^{k}$; see again [14, Chapter 12]. In the case in which $k=0$ we may identify $\varepsilon^{k}$ with $\mathbb{R}^{d}$, and $\Theta$ becomes the Lebesgue measure. A Poisson process $X$ on $\varepsilon^{k}$ (see, e.g. [4] for a definition of general Poisson processes) is stationary if and only if its intensity measure is a multiple of (2.3) for some $\mathbb{Q}$. Such stationary Poisson processes of $k$-flats are the subject of this paper. If $k=0$ then $X$ is just a stationary Poisson process on $\mathbb{R}^{d}$.

For any closed set $K \subset \mathbb{R}^{d}$, we define

$$
\Theta^{*}(K):=\Theta\left(\left\{F \in \mathcal{E}^{k}: F \cap K \neq \varnothing\right\}\right) .
$$

In the case in which $k=0$ this is just the volume of $K$. If $\mathbb{Q}$ is the uniform distribution and $K$ has some further properties, then this is the integral-geometric contents of $K$. If, for instance, $K$ is a compact and convex set then $\Theta^{*}(K)$ is proportional to the surface area of $K$ in the case in which $k=1$ and proportional to the mean breadth in the case in which $k=d-1$; see [14] and [15] for more details. For general $\mathbb{Q}$, we may interpret (2.4) as the generalized integral-geometric contents of $K$.

\subsection{Stopping sets}

Let $A \subset \mathcal{E}^{k}$ be measurable, and let $\mathcal{N}_{A}^{k}$ be the $\sigma$-field generated by the mapping $\eta \mapsto \eta \cap A$. A stopping set (defined on $N^{k}$ ) is a mapping $T: N^{k} \rightarrow \mathcal{F}\left(\mathcal{E}^{k}\right)$ such that

$$
\left\{\eta \in N^{k}: T(\eta) \subset K\right\} \in \mathcal{N}_{K}^{k}, \quad K \in \mathcal{F}\left(\varepsilon^{k}\right) .
$$

This is essentially the definition from [17]. Note, however, that we are not restricting $T$ to be compact. Moreover, owing to the special properties of the domain $N^{k}$, we can derive (see Appendix A) some more specific properties of a stopping set. The stopping $\sigma$-field associated with a stopping set $T$ is defined by

$$
\mathcal{N}_{T}^{k}:=\left\{A \in \mathcal{N}^{k}: A \cap\{T \subset K\} \in \mathcal{N}_{K}^{k} \text { for all } K \in \mathcal{F}\left(\mathcal{E}^{k}\right)\right\} .
$$


For the main purpose of this paper, it would be sufficient to concentrate on a generic type of stopping set constructed as follows. For any closed set $K \subset \mathbb{R}^{d}$, we define a measurable mapping $\pi_{K}^{\prime}: N^{k} \rightarrow N^{k}$ by

$$
\pi_{K}^{\prime}(\eta):=\{F \in \eta: F \cap K \neq \varnothing\} .
$$

If $T^{\prime}: N^{k} \rightarrow \mathcal{F}$ is measurable then $\mathcal{E}_{T^{\prime}}^{k}: N^{k} \rightarrow \mathcal{F}\left(\mathcal{E}^{k}\right)$ defined by

$$
\varepsilon_{T^{\prime}}^{k}(\eta):=\varepsilon_{T^{\prime}(\eta)}^{k}=\left\{F \in \varepsilon^{k}: F \cap T^{\prime}(\eta) \neq \varnothing\right\}
$$

is also measurable. We note that $\varepsilon_{T^{\prime}}^{k}(\eta)$ is closed if $T^{\prime}(\eta)$ is compact. It turns out that $\varepsilon_{T^{\prime}}^{k}$ is a stopping set if $T^{\prime}$ has the following natural stopping set property.

Proposition 2.1. Let $T^{\prime}: N^{k} \rightarrow \mathcal{F}$ be a measurable mapping satisfying

$$
\left\{T^{\prime} \subset K\right\} \in \sigma\left(\pi_{K}^{\prime}\right), \quad K \in \mathcal{F} .
$$

Assume that $\mathcal{E}_{T^{\prime}}^{k}$ is closed. Then $\mathcal{E}_{T^{\prime}}^{k}$ is a stopping set and

$$
\mathcal{N}_{\mathcal{E}_{T^{\prime}}^{k}}^{k}=\left\{A \in \mathcal{N}^{k}: A \cap\left\{T^{\prime} \subset K\right\} \in \sigma\left(\pi_{K}^{\prime}\right) \text { for all } K \in \mathcal{F}\right\} .
$$

The proof of this result and further details on a more general concept of a stopping set are given in Appendix A. If $k=0$ then $\varepsilon_{T^{\prime}}^{0}=T^{\prime}$. In this case the literature has numerous examples of stopping sets (see, e.g. [17]). Here is a simple example that applies for any $k$.

Example 2.1. Let $i \in \mathbb{N}$, and define a measurable mapping $\tau_{i}: N^{k} \rightarrow[0, \infty)$ by

$$
\tau_{i}(\eta):=\inf \left\{r \geq 0: \operatorname{card}\left(F \in \eta: F \cap r B^{d} \neq \varnothing\right) \geq i\right\} .
$$

It is easy to show that $T^{\prime}(\eta):=\tau_{i}(\eta) B^{d}$ (i.e. $T^{\prime}(\eta):=\mathbb{R}^{d}$ in the case where $\tau_{i}(\eta)=\infty$ ) gives a measurable mapping satisfying (2.7).

\subsection{Stationary random measures and mark distributions}

We work on the probability space $\left(N^{k}, \mathcal{N}^{k}, \mathrm{P}\right)$, where $\mathrm{P}$ is the distribution of a stationary Poisson process of $k$-flats with intensity $\gamma>0$. The identity on $N^{k}$ is denoted by $X$. A random measure $M$ on $\mathbb{R}^{d}$ (see, e.g. [4, Chapter 12]) is a random variable taking its values in the space $\boldsymbol{M}$ of all locally finite measures $\alpha$ on $\mathbb{R}^{d}$ equipped with the $\sigma$-field $\mathcal{M}$ generated by the mappings $\alpha \mapsto \alpha(B), B \in \mathcal{B}^{d}$. We write $M(\eta, B):=M(\eta)(B)$. Note that $N^{0}$ is a measurable subset of $\boldsymbol{M}$. An element of $\boldsymbol{N}^{0}$ is also called a simple counting measure on $\mathbb{R}^{d}$. A (simple) point process on $\mathbb{R}^{d}$ is a random measure $M$ satisfying $\mathrm{P}\left(M(X) \in N^{0}\right)=1$. A random measure $M$ on $\mathbb{R}^{d}$ is called stationary if

$$
M(\eta, B+x)=M(\eta-x, B), \quad \eta \in N^{k}, x \in \mathbb{R}^{d}, B \in \mathscr{B}^{d} .
$$

Here and later we use, for $A \subset \mathcal{E}^{k}, y \in \mathbb{R}^{d}$, and $c>0$, the notation $A+y:=\{F+y: F \in A\}$ and $c A:=\{c F: F \in A\}$. If $M$ is a stationary random measure then the distribution of $M(\cdot+x)$ is the same for any $x \in \mathbb{R}^{d}$.

Let $(\boldsymbol{X}, \mathcal{X})$ be a measurable space, and let $M_{\boldsymbol{X}}$ denote the space of all measures $\alpha$ on $\mathbb{R}^{d} \times \boldsymbol{X}$ such that $\alpha(\cdot \times \boldsymbol{X})$ is locally finite. The $\sigma$-field $\mathcal{M}_{\boldsymbol{X}}$ on $M_{\boldsymbol{X}}$ is defined exactly as above. A marked random measure $M$ on $\mathbb{R}^{d}$ with mark space $X$ is an $M_{X}$-valued random variable. 
It is called stationary if $M(\cdot \times C)$ is stationary for all $C \in \mathcal{X}$. If $M$ is such a stationary random measure then $\mathrm{E} M(B \times C)=\mathscr{H}^{d}(B) \mathbb{V}^{\prime}(C)$ for some measure $\mathbb{V}^{\prime}$ on $\boldsymbol{X}$. In case the intensity $\gamma_{M}:=\mathbb{V}^{\prime}(\boldsymbol{X})=\mathrm{E} M\left([0,1]^{d} \times \boldsymbol{X}\right)$ of $M$ is positive and finite we may normalize $\mathbb{V}^{\prime}$ to obtain the (Palm) mark distribution $\mathbb{V}$ of $M$. We then have the refined Campbell theorem

$$
\mathrm{E} \int f(x, y) M(X, \mathrm{~d}(x, y))=\gamma_{M} \iint f(x, y) \mathrm{d} x \mathbb{V}(\mathrm{d} y)
$$

for all measurable $f: \mathbb{R}^{d} \times \boldsymbol{X} \rightarrow[0, \infty)$, where $\mathrm{d} x$ means integration with respect to the Lebesgue measure $\mathscr{H}^{d}$. It is common to call $\mathbb{V}$ the distribution of the typical mark of $M$.

Let $N$ be a stationary random measure on $\mathbb{R}^{d}$. Then

$$
M(\eta, \cdot):=\int \mathbf{1}\{(x, \eta-x) \in \cdot\} N(\eta, \mathrm{d} x)
$$

is a stationary marked random measure with mark space $N^{k}$. If the intensity $\gamma_{N}:=\mathrm{E} N\left([0,1]^{d}\right)$ of $N$ is positive and finite, then the mark distribution of this $M$ is the Palm probability measure $\mathrm{P}_{N}^{0}$ of $N$. Note that this is a distribution on $N^{k}$. The refined Campbell theorem takes the form

$$
\mathrm{E} \int f(X-x, x) N(X, \mathrm{~d} x)=\gamma_{N} \iint f(\eta, x) \mathrm{d} x \mathrm{P}_{N}^{0}(\mathrm{~d} \eta)
$$

for all measurable $f: N^{k} \times \mathbb{R}^{d} \rightarrow[0, \infty)$. The measure $\mathrm{P}_{N}^{0}$ is describing the statistical behavior of $X$ as seen from a typical point of $N$.

\section{Formulation and proof of the main result}

For the remainder of the paper, we let $\left(N^{k}, \mathcal{N}^{k}, \mathrm{P}\right)$ be the underlying probability space; see Subsection 2.4.

We consider an integer $n \geq 0$, measurable mappings $S:\left(\mathcal{E}^{k}\right)^{n} \times \mathbb{R}^{d} \times N^{k} \rightarrow \mathcal{F}\left(\mathcal{E}^{k}\right)$ and $R:\left(\mathcal{E}^{k}\right)^{n} \times \mathbb{R}^{d} \times N^{k} \rightarrow[0, \infty)$, and a kernel $\mu$ from $\left(\mathcal{E}^{k}\right)^{n}$ to $\mathbb{R}^{d}$ with the following properties. The mapping $S\left(F_{1}, \ldots, F_{n}, x, \cdot\right)$ is assumed to be a stopping set for all $F_{1}, \ldots, F_{n} \in \mathcal{E}^{k}$ and $x \in \mathbb{R}^{d}$. Accordingly, we define $\mathcal{N}_{F_{1}, \ldots, F_{n}, x}^{k}$ as the associated stopping $\sigma$-field. Furthermore, we assume that, for all $F_{1}, \ldots, F_{n} \in \mathcal{E}^{k}, x, y \in \mathbb{R}^{d}$, and $\eta \in N^{k}$, the equivariance property

$$
S\left(F_{1}+y, \ldots, F_{n}+y, x+y, \eta+y\right)=S\left(F_{1}, \ldots, F_{n}, x, \eta\right)+y
$$

holds. If $n=0, \mu$ is assumed to be the Lebesgue measure on $\mathbb{R}^{d}$. Otherwise, $\mu$ is assumed to satisfy the equivariance property

$$
\mu\left(F_{1}+y, \ldots, F_{n}+y, B\right)=\mu\left(F_{1}, \ldots, F_{n}, B-y\right)
$$

for all $F_{1}, \ldots, F_{n} \in \mathcal{E}^{k}, y \in \mathbb{R}^{d}$, and $B \in \mathscr{B}^{d}$. The mapping $R$ has to satisfy the invariance property

$$
R\left(F_{1}+y, \ldots, F_{n}+y, x+y, \eta+y\right)=R\left(F_{1}, \ldots, F_{n}, x, \eta\right),
$$

and $R\left(F_{1}, \ldots, F_{n}, x, \cdot\right)$ is assumed to be $\mathcal{N}_{F_{1}, \ldots, F_{n}, x}^{k}$-measurable for all $F_{1}, \ldots, F_{n} \in \mathcal{E}^{k}$ and $x \in \mathbb{R}^{d}$. Let $m \geq 0$, and define

$$
R_{m}\left(F_{1}, \ldots, F_{n}, x, \eta\right):=\mathbf{1}\left\{\eta\left(S\left(F_{1}, \ldots, F_{n}, x, \eta\right)\right)=m\right\} R\left(F_{1}, \ldots, F_{n}, x, \eta\right)
$$


for $F_{1}, \ldots, F_{n} \in \mathcal{E}^{k}, x \in \mathbb{R}^{d}$, and $\eta \in N^{k}$. We assume that, for all $c>0$, the following scaling relation holds:

$$
\begin{gathered}
\int \mathbf{1}\left\{x \in c^{-1}[0,1]^{d}\right\} \mathrm{E} R_{m}\left(F_{1}, \ldots, F_{n}, x, X\right) \mu\left(F_{1}, \ldots, F_{n}, \mathrm{~d} x\right) \\
=c^{-j} \int \mathbf{1}\left\{x \in[0,1]^{d}\right\} \mathrm{E} R_{m}\left(c F_{1}, \ldots, c F_{n}, x, c X\right) \\
\times \mu\left(c F_{1}, \ldots, c F_{n}, \mathrm{~d} x\right), \quad F_{1}, \ldots, F_{n} \in \mathcal{E}^{k}
\end{gathered}
$$

for some $j \in\{0, \ldots, d\}$.

Remark 3.1. Consider the case in which $n \geq 1$, and let $Z:\left(\mathcal{E}^{k}\right)^{n} \rightarrow \mathcal{F}$ be a measurable mapping. A possible choice of the kernel $\mu$ is

$$
\mu\left(F_{1}, \ldots, F_{n}, \cdot\right):=\int \mathbf{1}\left\{x \in Z\left(F_{1}, \ldots, F_{n}\right) \cap \cdot\right\} \mathcal{H}^{j}(\mathrm{~d} x) .
$$

Assuming the above to be locally finite for all $F_{1}, \ldots, F_{n} \in \mathcal{E}^{k}$, Corollary 2.1.4 of [16] implies that $\mu$ is a kernel. If $Z$ is equivariant under translations then $\mu$ satisfies (3.2). The scaling relation (3.5) is implied by

$$
\begin{aligned}
& \mathrm{E} R_{m}\left(c F_{1}, \ldots, c F_{n}, c x, c X\right) \mathbf{1}\left\{c x \in Z\left(c F_{1}, \ldots, c F_{n}\right)\right\} \\
&= \mathrm{E} R_{m}\left(F_{1}, \ldots, F_{n}, x, X\right) \\
& \times \mathbf{1}\left\{x \in Z\left(F_{1}, \ldots, F_{n}\right)\right\}, \quad F_{1}, \ldots, F_{n} \in \mathcal{E}^{k}, x \in \mathbb{R}^{d}, c>0 .
\end{aligned}
$$

This holds, for instance, if $S$ and $Z$ are equivariant under scaling and $R$ is invariant under scaling.

Remark 3.2. Assume that the kernel $\mu$ satisfies the scaling relation (1.2) and that

$$
\begin{aligned}
& \mathrm{E} R_{m}\left(c F_{1}, \ldots, c F_{n}, c x, c X\right) \\
& \quad=\mathrm{E} R_{m}\left(F_{1}, \ldots, F_{n}, x, X\right), \quad F_{1}, \ldots, F_{n} \in \mathcal{E}^{k}, x \in \mathbb{R}^{d}, c>0 .
\end{aligned}
$$

Then (3.5) holds.

Recalling (2.3), we now define, for any $\eta \in N^{k}$, a measure $M(\eta, \cdot)$ on $\mathbb{R}^{d} \times[0, \infty]$ by

$$
\begin{aligned}
M(\eta, B \times C):=\iint & \mathbf{1}\left\{x \in B, \Theta\left(S\left(F_{1}, \ldots, F_{n}, x, \eta_{F_{1}, \ldots, F_{n}}\right)\right) \in C\right\} \\
& \times R_{m}\left(F_{1}, \ldots, F_{n}, x, \eta_{F_{1}, \ldots, F_{n}}\right) \\
& \times \mu\left(F_{1}, \ldots, F_{n}, \mathrm{~d} x\right) \eta^{(n)}\left(\mathrm{d}\left(F_{1}, \ldots, F_{n}\right)\right),
\end{aligned}
$$

where $\eta_{F_{1}, \ldots, F_{n}}^{!}:=\eta \backslash\left\{F_{1}, \ldots, F_{n}\right\}$ and $\eta^{(n)}$ is the measure on $\left(\mathcal{E}^{k}\right)^{n}$ such that integration with respect to this measure is just summation over all tuples $\left(F_{1}, \ldots, F_{n}\right) \in \eta^{n}$ with pairwise different entries. In the case in which $n=0$ this has to be interpreted as

$$
M(\eta, B \times C):=\int_{B} 1\{\Theta(S(x, \eta)) \in C\} R_{m}(x, \eta) \mathrm{d} x .
$$

We assume that $M(\eta, \cdot \times[0, \infty])$ is locally finite. Then $M \equiv M(\cdot, \cdot)$ is clearly a marked random measure, and the equivariance and invariance assumptions, (3.1)-(3.3), easily imply that $M$ is stationary in the sense of Subsection 2.4. The proof of Theorem 3.1, below, implies that the intensity $\gamma_{M}$ of $M$ is given by (1.1). 
Theorem 3.1. Assume that $M$ defined by (3.7) has a positive and finite intensity, and that $m+n-(d-j) /(d-k)>0$. Then the mark distribution of $M$ is $\Gamma(m+n-(d-j) /(d-k), \gamma)$, where $j=d$ in the $n=0$ case.

Remark 3.3. For formal reasons, we have defined $M$ as a random measure on $\mathbb{R}^{d} \times[0, \infty]$. But, if $0<\gamma_{M}<\infty$ then Theorem 3.1 implies in particular that $M$ is almost surely concentrated on $\mathbb{R}^{d} \times(0, \infty)$. Indeed, we then have $\mathrm{E} M(B \times\{0\})=\mathrm{E} M(B \times\{\infty\})=0$ for any bounded (and, hence, any) Borel set $B \subset \mathbb{R}^{d}$. In particular, there is no need to exclude the case $\Theta\left(S\left(F_{1}, \ldots, F_{n}, x, \eta_{F_{1}, \ldots, F_{n}}\right)\right)=0$ in the definition of $M,(3.7)$, explicitly.

Remark 3.4. Let $\tilde{S}:\left(\mathcal{E}^{k}\right)^{n} \times \mathbb{R}^{d} \times \boldsymbol{N}^{k} \rightarrow \mathcal{F}$ be measurable, and define $S:=\mathcal{E}_{\tilde{S}}^{k}$ as in (2.6) and assume that $S$ is closed. Equivariance of $S$ under translation is then equivalent to the corresponding equivariance of $\tilde{S}$. Similar statements apply to the equivariance and invariance of $S$ under scaling. The random measure $M$ takes the form

$$
\begin{aligned}
M(\eta, B \times C):=\iint & \mathbf{1}\left\{x \in B, \Theta^{*}\left(\tilde{S}\left(F_{1}, \ldots, F_{n}, x, \eta_{F_{1}, \ldots, F_{n}}^{!}\right)\right) \in C\right\} \\
& \times R_{m}\left(F_{1}, \ldots, F_{n}, x, \eta_{F_{1}, \ldots, F_{n}}\right) \mu\left(F_{1}, \ldots, F_{n}, \mathrm{~d} x\right) \eta^{(n)}\left(\mathrm{d}\left(F_{1}, \ldots, F_{n}\right)\right),
\end{aligned}
$$

where $\Theta^{*}$, the generalized integral-geometric contents, is defined in (2.4). By Proposition 2.1, the stopping set property of $S$ holds if $\tilde{S}\left(F_{1}, \ldots, F_{n}, x, \cdot\right)$ has the property (2.7) for all $F_{1}, \ldots, F_{n} \in N^{k}$ and $x \in \mathbb{R}^{d}$.

To state a second result, we consider a measurable mapping $G$ on $\left(\mathcal{E}^{k}\right)^{n} \times N^{k}$ taking values in some measurable space $(\boldsymbol{X}, \mathcal{X})$. We assume that $G$ is invariant under scaling. For any $\eta \in \boldsymbol{N}^{k}, B \in \mathscr{B}^{d}$, and measurable $D \subset[0, \infty] \times \boldsymbol{X}$, we define

$$
\begin{aligned}
M_{G}(\eta, B \times D):=\iint & \mathbf{1}\left\{\left(\Theta\left(S\left(F_{1}, \ldots, F_{n}, x, \eta_{F_{1}, \ldots, F_{n}}\right)\right),\right.\right. \\
& \left.\left.G\left(F_{1}-x, \ldots, F_{n}-x, \eta_{F_{1}, \ldots, F_{n}}-x\right)\right) \in D\right\} \\
\times & \mathbf{1}\{x \in B\} R_{m}\left(F_{1}, \ldots, F_{n}, x, \eta_{F_{1}, \ldots, F_{n}}\right) \mu\left(F_{1}, \ldots, F_{n}, \mathrm{~d} x\right) \\
\times & \eta^{(n)}\left(\mathrm{d}\left(F_{1}, \ldots, F_{n}\right)\right),
\end{aligned}
$$

with the obvious interpretation in the $n=0$ case; cf. (3.8). Again, we obtain a stationary marked random measure $M_{G}$, this time with mark space $[0, \infty] \times X$. Note the equality $M(B \times C)=$ $M_{G}(B \times(C \times X))$. The following result generalizes the independence assertions of Theorems 3 and 4 of [12].

Theorem 3.2. Under the hypothesis of Theorem 3.1, the mark distribution of $M_{G}$ is a product of a gamma distribution and a distribution on $\boldsymbol{X}$.

In proving our results we extend and unify ideas from [12] and [17]. Moreover, we will use some specific properties of stopping sets defined on $N^{k}$ which are listed in Appendix A.

First we recall that the identity $X$ on $N^{k}$ is a Poisson process of $k$-flats with intensity measure $\gamma \Theta$. For any $\rho>0$, we let $\mathrm{P}_{\rho}$ denote the distribution of a Poisson process of $k$-flats with intensity measure $\rho \Theta$. The expectation with respect to $\mathrm{P}_{\rho}$ is denoted by $\mathrm{E}_{\rho}$. We have the useful scaling property

$$
\mathrm{P}_{\rho}(\cdot)=\mathrm{P}_{1}\left(\rho^{-1 /(d-k)} X \in \cdot\right) .
$$


Indeed, by the well-known mapping theorem, $\rho^{-1 /(d-k)} X$ is a Poisson process under $\mathrm{P}_{1}$, and an easy computation shows that its intensity measure is given by $\rho \Theta$. Note that $\mathrm{P}=\mathrm{P}_{\gamma}$. The following result is an analogue of Theorem 1 of [12] and Equation (9) of [17]. We recall that $X(B)=\operatorname{card}(X \cap B)$ for a Borel set $B \subset \mathbb{R}^{d}$. If $T: N^{k} \rightarrow \mathcal{F}\left(\mathcal{E}^{k}\right)$ is measurable then $X(T)$ is the random variable $\operatorname{card}(X \cap T)$.

Proposition 3.1. Let $T: N^{k} \rightarrow \mathcal{F}\left(\mathcal{E}^{k}\right)$ be a stopping set, and let $\rho>0$. Then we have, for all measurable $g: N^{k} \rightarrow[0, \infty)$,

$$
\mathrm{E}_{\rho} \mathbf{1}\{X(T)=m\} g(X \cap T)=\frac{\rho^{m}}{\gamma^{m}} \mathrm{E}[\exp [(\gamma-\rho) \Theta(T)] \mathbf{1}\{X(T)=m\} g(X \cap T)] .
$$

Proof. Satz 3.1 of [7] says that

$$
\mathrm{E} \int h(X \backslash\{F\}, F) X(\mathrm{~d} F)=\mathrm{E} \int h(X, F) \Theta(\mathrm{d} F)
$$

for all measurable $h: \boldsymbol{N}^{k} \times \mathcal{E}^{k} \rightarrow[0, \infty)$. Assume that $m \geq 1$, and let $f: \boldsymbol{N}^{k} \times \mathcal{F}\left(\mathcal{E}^{k}\right) \rightarrow$ $[0, \infty)$ be measurable. Using Lemma A.3 along with an iterated version of (3.11), we obtain

$$
\begin{aligned}
& \operatorname{Ex} f(X \cap T, T) \mathbf{1}\{X(T)=m\} \\
&=\frac{\gamma^{m}}{m !} \int f\left(\left\{F_{1}, \ldots, F_{m}\right\}, T\left(\left\{F_{1}, \ldots, F_{m}\right\}\right)\right) \exp \left[-\gamma \Theta\left(T\left(\left\{F_{1}, \ldots, F_{m}\right\}\right)\right)\right] \\
& \times \mathbf{1}\left\{F_{1}, \ldots, F_{m} \in T\left(\left\{F_{1}, \ldots, F_{m}\right\}\right)\right\} \Theta^{m}\left(\mathrm{~d}\left(F_{1}, \ldots, F_{m}\right)\right) .
\end{aligned}
$$

In the $m=0$ case we have instead

$$
\mathrm{E} f(X \cap T, T) \mathbf{1}\{X(T)=0\}=f(\varnothing, T(\varnothing)) \exp [-\gamma \Theta(T(\varnothing)] .
$$

Applying (3.12) with $\rho$ instead of $\gamma$ yields

$$
\begin{aligned}
\mathrm{E}_{\rho} \mathbf{1}\{X(T)=m\} g(X \cap T) & \\
=\frac{\rho^{m}}{\gamma^{m}} \frac{\gamma^{m}}{m !} \int & g\left(\left\{F_{1}, \ldots, F_{m}\right\}\right) \exp \left[-\gamma \Theta\left(T\left(\left\{F_{1}, \ldots, F_{m}\right\}\right)\right)\right] \\
& \times \mathbf{1}\left\{\Theta\left(T\left(\left\{F_{1}, \ldots, F_{m}\right\}\right)\right)<\infty\right\} \exp \left[-(\rho-\gamma) \Theta\left(T\left(\left\{F_{1}, \ldots, F_{m}\right\}\right)\right)\right] \\
& \times \mathbf{1}\left\{F_{1}, \ldots, F_{m} \in T\left(\left\{F_{1}, \ldots, F_{m}\right\}\right)\right\} \Theta^{m}\left(\mathrm{~d}\left(F_{1}, \ldots, F_{m}\right)\right) .
\end{aligned}
$$

Another application of (3.12) gives

$$
\begin{aligned}
B E_{\rho} & \mathbf{1}\{X(T)=m\} g(X \cap T) \\
= & \frac{\rho^{m}}{\gamma^{m}} \mathrm{E}[\mathbf{1}\{X(T)=m\} g(X \cap T) \mathbf{1}\{\Theta(T)<\infty\} \exp [-(\rho-\gamma) \Theta(T)]] .
\end{aligned}
$$

As (3.12) also implies that

$$
\mathrm{P}(\Theta(T)=\infty, X(T)=m)=0,
$$

we obtain the assertion for $m \geq 1$. In the $m=0$ case the assertion follows in the same way from (3.13). 
Proof of Theorem 3.1. We prove the theorem for the case in which $n \geq 1$. The proof in the $n=0$ case is similar but simpler. Let $\mathbb{V}$ be the mark distribution of $M$. Take $s<\gamma$, and define $\rho:=(\gamma-s) / \gamma$ and $C_{\rho}:=\rho^{-1 /(d-k)}[0,1]^{d}$. Let

$$
L(s):=\int \exp [s t] \mathbb{V}(\mathrm{d} t)
$$

be the moment generating function of $\mathbb{V}$ evaluated at $s$. From the definition of $M,(3.7)$, and the refined Campbell theorem, (2.9), we have

$$
\begin{aligned}
\gamma_{M} L(s)=\rho^{d /(d-k)} \mathrm{E} \iint & \exp \left[s \Theta\left(S\left(F_{1}, \ldots, F_{n}, x, X_{F_{1}, \ldots, F_{n}}^{!}\right)\right] R_{m}\left(F_{1}, \ldots, F_{n}, x, X_{F_{1}, \ldots, F_{n}}\right)\right. \\
& \times \mathbf{1}\left\{x \in C_{\rho}\right\} \mu\left(F_{1}, \ldots, F_{n}, \mathrm{~d} x\right) X^{(n)}\left(\mathrm{d}\left(F_{1}, \ldots, F_{n}\right)\right) .
\end{aligned}
$$

From the multivariate version of Mecke's formula we obtain

$$
\begin{aligned}
\gamma_{M} L(s)=\rho^{d /(d-k)} \gamma^{n} \mathrm{E} \iint & \exp \left[s \Theta\left(S\left(F_{1}, \ldots, F_{n}, x, X\right)\right)\right] R_{m}\left(F_{1}, \ldots, F_{n}, x, X\right) \\
& \times \mathbf{1}\left\{x \in C_{\rho}\right\} \mu\left(F_{1}, \ldots, F_{n}, \mathrm{~d} x\right) \Theta^{n}\left(\mathrm{~d}\left(F_{1}, \ldots, F_{n}\right)\right) .
\end{aligned}
$$

Since the kernel $\mu$ is $\sigma$-finite, we can apply Fubini's theorem to obtain

$$
\begin{aligned}
& \gamma_{M} L(s)=\rho^{d /(d-k)} \gamma^{n} \iint \mathrm{E}\left[\exp \left[s \Theta\left(S\left(F_{1}, \ldots, F_{n}, x, X\right)\right)\right] R_{m}\left(F_{1}, \ldots, F_{n}, x, X\right)\right] \\
& \times \mathbf{1}\left\{x \in C_{\rho}\right\} \mu\left(F_{1}, \ldots, F_{n}, \mathrm{~d} x\right) \Theta^{n}\left(\mathrm{~d}\left(F_{1}, \ldots, F_{n}\right)\right) .
\end{aligned}
$$

We have assumed that $S\left(F_{1}, \ldots, F_{n}, x, \cdot\right)$ is a stopping set for all $F_{1}, \ldots, F_{n} \in \mathcal{E}^{k}$ and $x \in \mathbb{R}^{d}$. Moreover, we have, from Lemma A.2(i),

$$
R\left(F_{1}, \ldots, F_{n}, x, X\right)=R\left(F_{1}, \ldots, F_{n}, x, X \cap S\left(F_{1}, \ldots, F_{n}, x, X\right)\right),
$$

since $R\left(F_{1}, \ldots, F_{n}, x, \cdot\right)$ is assumed to be $\mathcal{N}_{F_{1}, \ldots, F_{n}, x}^{k}$-measurable. Taking into account the definition of $R_{m},(3.4)$, we obtain, from Proposition 3.1 and (3.14),

$$
\begin{aligned}
& \gamma_{M} L(s)=\rho^{d /(d-k)} \gamma^{n} \frac{\gamma^{m}}{\rho^{m} \gamma^{m}} \iint \mathrm{E}_{\rho \gamma}[ \exp \left[(\rho \gamma-\gamma+s) \Theta\left(S\left(F_{1}, \ldots, F_{n}, x, X\right)\right)\right] \\
&\left.\times R_{m}\left(F_{1}, \ldots, F_{n}, x, X\right)\right] \\
& \times \mathbf{1}\left\{x \in C_{\rho}\right\} \mu\left(F_{1}, \ldots, F_{n}, \mathrm{~d} x\right) \Theta^{n}\left(\mathrm{~d}\left(F_{1}, \ldots, F_{n}\right)\right) .
\end{aligned}
$$

Since $\rho \gamma=\gamma-s$, this simplifies to

$$
\begin{aligned}
\gamma_{M} L(s)=\rho^{d /(d-k)} \frac{\gamma^{n}}{\rho^{m}} \iint & \mathrm{E}_{\rho \gamma}\left[R_{m}\left(F_{1}, \ldots, F_{n}, x, X\right)\right] \mathbf{1}\left\{x \in \rho^{-1 /(d-k)}[0,1]^{d}\right\} \\
& \times \mu\left(F_{1}, \ldots, F_{n}, \mathrm{~d} x\right) \Theta^{n}\left(\mathrm{~d}\left(F_{1}, \ldots, F_{n}\right)\right) .
\end{aligned}
$$

The scaling property (3.10) implies that $\mathrm{P}_{\rho \gamma}=\mathrm{P}\left(\rho^{-1 /(d-k)} X \in \cdot\right)$. Using the scale invariance (3.5), we arrive at

$$
\begin{aligned}
\gamma_{M} L(s)= & \rho^{d /(d-k)} \frac{\gamma^{n}}{\rho^{m}} \rho^{-j /(d-k)} \\
& \times \iint \mathrm{E}\left[R_{m}\left(\rho^{1 /(d-k)} F_{1}, \ldots, \rho^{1 /(d-k)} F_{n}, x, X\right)\right] \\
& \times \mathbf{1}\left\{x \in[0,1]^{d}\right\} \mu\left(\rho^{1 /(d-k)} F_{1}, \ldots, \rho^{1 /(d-k)} F_{n}, \mathrm{~d} x\right) \Theta^{n}\left(\mathrm{~d}\left(F_{1}, \ldots, F_{n}\right)\right) .
\end{aligned}
$$


Recalling definition (2.3) of $\Theta$, this means that

$$
\begin{aligned}
\gamma_{M} L(s)=\rho^{(d-j) /(d-k)} \frac{\gamma^{n}}{\rho^{m}} \\
\times \iiint \mathrm{E}\left[R_{m}\left(F_{1}+\rho^{1 /(d-k)} x_{1}, \ldots, F_{n}+\rho^{1 /(d-k)} x_{n}, \rho^{1 /(d-k)} x, X\right)\right] \\
\quad \times \mathbf{1}\left\{x \in[0,1]^{d}\right\} \mu\left(F_{1}+\rho^{1 /(d-k)} x_{1}, \ldots, F_{n}+\rho^{1 /(d-k)} x_{n}, \mathrm{~d} x\right) \\
\quad \times \mathbf{1}\left\{x_{1} \in F_{1}^{\perp}, \ldots, x_{n} \in F_{n}^{\perp}\right\}\left(\mathcal{H}^{d-k}\right)^{n}\left(\mathrm{~d}\left(x_{1}, \ldots, x_{n}\right)\right) \mathbb{Q}^{n}\left(\mathrm{~d}\left(F_{1}, \ldots, F_{n}\right)\right) .
\end{aligned}
$$

For any fixed linear subspaces $F_{1}, \ldots, F_{n}$, the transformation

$$
\left(y_{1}, \ldots, y_{n}\right):=\rho^{1 /(d-k)}\left(x_{1}, \ldots, x_{n}\right)
$$

has the Jacobian $\rho^{-n(d-k) /(d-k)}=\rho^{-n}$. Therefore,

$$
\gamma_{M} L(s)=\gamma^{n} \rho^{(d-j) /(d-k)} \rho^{-(m+n)} a,
$$

where

$$
a:=\iint \mathrm{E}\left[R_{m}\left(F_{1}, \ldots, F_{n}, y, X\right)\right] \mathbf{1}\left\{y \in[0,1]^{d}\right\} \mu\left(F_{1}, \ldots, F_{n}, \mathrm{~d} y\right) \Theta^{n}\left(\mathrm{~d}\left(F_{1}, \ldots, F_{n}\right)\right) .
$$

Setting $s=0$ yields $\rho=1$ and, therefore, $\gamma_{M}=\gamma^{n} a$, i.e. (1.1). It follows that

$$
L(s)=\rho^{-(m+n-(d-j) /(d-k))}=\left(\frac{\gamma}{\gamma-s}\right)^{m+n-(d-j) /(d-k)},
$$

which is the moment generating function of $\Gamma(m+n-(d-j) /(d-k), \gamma)$.

Proof of Theorem 3.2. Let $\mathbb{W}$ be the mark distribution of $M_{G}$, and let $f: \boldsymbol{X} \rightarrow[0, \infty)$ be measurable. Replacing $R_{m}$ in the above proof by the translation and scale invariant function $R_{m}\left(F_{1}, \ldots, F_{n}, x, \eta\right) f\left(G\left(F_{1}-x, \ldots, F_{n}-x, \eta-x\right)\right)$ we obtain, exactly as before,

$$
\gamma_{M} \int \exp [s t] f(z) \mathbb{W}(\mathrm{d}(t, z))=\gamma^{n} \rho^{-(m+n-(d-j) /(d-k))} a_{f},
$$

where

$$
\begin{aligned}
a_{f}:=\iint & \mathrm{E}\left[R_{m}\left(F_{1}, \ldots, F_{n}, y, X\right) f\left(G\left(F_{1}-y, \ldots, F_{n}-y, X-y\right)\right)\right] \\
& \times \mathbf{1}\left\{y \in[0,1]^{d}\right\} \mu\left(F_{1}, \ldots, F_{n}, \mathrm{~d} y\right) \Theta^{n}\left(\mathrm{~d}\left(F_{1}, \ldots, F_{n}\right)\right) .
\end{aligned}
$$

The choice $s=0$ yields

$$
\gamma_{M} \int f(z) \mathbb{W}(\mathrm{d}(t, z))=\gamma^{n} a_{f}
$$

Combining this with (3.15) and (3.16) gives

$$
\int \exp [s t] f(z) \mathbb{W}(\mathrm{d}(t, z))=\int \exp [s t] \mathbb{W}(\mathrm{d}(t, z)) \int f(z) \mathbb{W}(\mathrm{d}(t, z)) .
$$

This is sufficient for concluding the assertion. 
Remark 3.5. Assume that $S$ does not depend on the last argument. Then the measurability assumptions on $S$ can be weakened. Indeed, the above proof shows that it is sufficient to assume that $\left(F_{1}, \ldots, F_{n}, x, F\right) \mapsto \mathbf{1}\left\{F \in S\left(F_{1}, \ldots, F_{n}, x\right)\right\}$ is measurable. In particular, $S\left(F_{1}, \ldots, F_{n}, x\right)$ need not be closed.

In the remainder of this section we give an alternative, slightly more succinct, formulation of the above theorems. To do so, we need to assume the existence of measurable mappings $g_{1}, \ldots, g_{n}: N^{k} \rightarrow \mathcal{E}^{k}$ such that, for all $\eta \in N^{k}$ and all pairwise different $F_{1}, \ldots, F_{n} \in \eta$, we have

$$
\left\{g_{1}(\eta-x)+x, \ldots, g_{n}(\eta-x)+x\right\}=\left\{F_{1}, \ldots, F_{n}\right\}
$$

for $\mu\left(F_{1}, \ldots, F_{n}, \cdot\right)$-almost every (a.e.) $x \in \mathbb{R}^{d}$ with $R_{m}\left(F_{1}, \ldots, F_{n}, x, \eta_{F_{1}, \ldots, F_{n}}^{!}\right)>0$. This says that, for all 'interesting' tuples $\left(F_{1}, \ldots, F_{n}, x, \eta\right)$, the flats $F_{1}, \ldots, F_{n}$ are determined by $\eta$ and $x$ in a measurable and translation-equivariant way. Using these functions, we define

$$
S^{*}(\eta):=S\left(g_{1}(\eta), \ldots, g_{n}(\eta), 0, \eta \backslash\left\{g_{1}(\eta), \ldots, g_{n}(\eta)\right\}\right), \quad \eta \in N^{k} .
$$

We also consider a measurable scale-invariant mapping $G$ on $\left(\varepsilon^{k}\right)^{n} \times N^{k}$ taking values in some measurable space $(\boldsymbol{X}, \mathcal{X})$. Let

$$
G^{*}(\eta):=G\left(g_{1}(\eta), \ldots, g_{n}(\eta), \eta \backslash\left\{g_{1}(\eta), \ldots, g_{n}(\eta)\right\}\right), \quad \eta \in N^{k} .
$$

Finally, we define a stationary random measure $M^{\prime}$ by $M^{\prime}(\eta, B):=M(\eta, B \times[0, \infty])$, i.e.

$$
\begin{aligned}
M^{\prime}(\eta, B):=\iint & \mathbf{1}\{x \in B\} R_{m}\left(F_{1}, \ldots, F_{n}, x, \eta_{F_{1}, \ldots, F_{n}}\right) \\
& \times \mu\left(F_{1}, \ldots, F_{n}, \mathrm{~d} x\right) \eta^{(n)}\left(\mathrm{d}\left(F_{1}, \ldots, F_{n}\right)\right) .
\end{aligned}
$$

Theorem 3.3. Let the above assumptions and the assumptions of Theorem 3.1 be satisfied. Then $\Theta\left(S^{*}\right)$ and $G^{*}$ are independent under the Palm probability measure $\mathrm{P}_{M^{\prime}}^{0}$ and $\Theta\left(S^{*}\right)$ has $a \Gamma(m+n-(d-j) /(d-k), \gamma)$-distribution, where $j=d$ in the $n=0$ case.

Proof. From (3.17) we can easily show that the random measure $M_{G}$ defined by (3.9) can be written as

$$
M_{G}(\eta, B \times C)=\int \mathbf{1}\{x \in B\} \mathbf{1}\left\{\left(\Theta\left(S^{*}(\eta-x)\right), G^{*}(\eta-x)\right) \in C\right\} M^{\prime}(\eta, \mathrm{d} x) .
$$

Hence, the refined Campbell theorem, (2.10), shows that $\mathrm{P}_{M^{\prime}}^{0}\left(\left(\Theta\left(S^{*}(X)\right), G^{*}(X)\right) \in \cdot\right)$ is the mark distribution of $M_{G}$, and the assertions follow from Theorems 3.1 and 3.2.

Remark 3.6. The results of this paper can be generalized to independently marked Poisson processes of flats as follows. Let $(X, \mathcal{X})$ be a measurable space, and let $N_{X}^{k}$ be the space of all counting measures $\psi$ on $\varepsilon^{k} \times \boldsymbol{X}$ such that $\psi(\cdot \times \boldsymbol{X})$ is a locally finite simple counting measure. The space $N_{\boldsymbol{X}}^{k}$ can be equipped with a $\sigma$-field $\mathcal{N}_{\boldsymbol{X}}^{k}$ as before. On $\boldsymbol{N}_{\boldsymbol{X}}^{k}$ we may then consider the distribution of a Poisson process on $\mathcal{E}^{k} \times X$ with intensity measure $\gamma \Theta \otimes \mathbb{L}$, where $\mathbb{L}$ is a distribution on $\boldsymbol{X}$. A stopping set is now a measurable mapping $T: N_{X}^{k} \rightarrow \mathcal{F}\left(\mathcal{E}^{k}\right)$ such that

$$
\left\{\psi \in N_{X}^{k}: T(\psi) \subset K\right\} \in \mathcal{N}_{K}^{k}, \quad K \in \mathcal{F}\left(\mathcal{E}^{k}\right),
$$

where $\mathcal{N}_{K}^{k}:=\sigma\left(\pi_{K}\right)$ and $\pi_{K}(\psi):=\{(F, z) \in \psi: F \in K\}$. The results in Appendix A apply in this setting, and the above theorems can be extended in the obvious way. One example of potential interest (e.g. in mobile telecommunication) is a Poisson process $X$ of lines, with each line carrying an independent Poisson point process. 


\section{Some special cases}

In this section we will show how the classical gamma-type results can be derived from our general theorems.

\subsection{Miles' complementary theorem}

In this subsection we assume that $S$ and $R$ do not depend on the last argument, and that $j=0$ and $n \geq 1$. Under the assumptions of Theorem 3.1 (in particular (3.5)), the typical mark of the stationary marked random measure $M$ defined by (3.7) has a $\Gamma(m+n-d /(d-k), \gamma)$ distribution. This is a slight generalization of the so-called complementary theorem derived in [8]; see also [12]. In its original formulation, $S$ is given as in Remark $3.4, R \equiv 1$, and $\mu\left(F_{1}, \ldots, F_{n}, \cdot\right)$ is the Dirac measure at some point $z\left(F_{1}, \ldots, F_{n}\right)$. The centroid $z:\left(\varepsilon^{k}\right)^{n} \rightarrow$ $\mathbb{R}^{d}$ is assumed measurable and equivariant with respect to translation and scaling. Taking a measurable and scale-invariant mapping $G$ on $\left(\varepsilon^{k}\right)^{n} \times N^{k}$, the random marked measure (3.9) takes the form

$$
\begin{aligned}
M_{G}(\eta, B \times \cdot):=\int & \mathbf{1}\left\{z\left(F_{1}, \ldots, F_{n}\right) \in B\right\} \mathbf{1}\left\{\left(\Theta\left(S^{\prime}\left(F_{1}, \ldots, F_{n}\right)\right), G^{\prime}\left(F_{1}, \ldots, F_{n}\right)\right) \in \cdot\right\} \\
& \times \mathbf{1}\left\{\eta\left(S^{\prime}\left(F_{1}, \ldots, F_{n}\right)\right)=m\right\} \eta^{(n)}\left(\mathrm{d}\left(F_{1}, \ldots, F_{n}\right)\right),
\end{aligned}
$$

where $S^{\prime}\left(F_{1}, \ldots, F_{n}\right):=S\left(F_{1}, \ldots, F_{n}, z\left(F_{1}, \ldots, F_{n}\right)\right)$ and

$$
G^{\prime}\left(F_{1}, \ldots, F_{n}\right):=G\left(F_{1}-z\left(F_{1}, \ldots, F_{n}\right), \ldots, F_{n}-z\left(F_{1}, \ldots, F_{n}\right)\right) .
$$

The scale invariance, (3.5), holds if $S$ (and, hence, also $S^{\prime}$ ) is equivariant under scaling. By Theorem 3.2, the mark distribution of $M_{G}$ is a product measure. The papers [2], [8], and [12] present many examples where the complementary theorem applies. In fact, Examples 1.3 and 1.5 also belong to this category. Example 1.2 is based on a simple extension of Miles' theorem to the case in which $R \not \equiv 1$.

\subsection{Subprocesses}

Again, we will consider a case where $S$ and $R$ do not depend on the last argument. Let $H \in \mathcal{N}^{k}$ and $S^{\prime}: \boldsymbol{N}^{k} \rightarrow \mathcal{F}\left(\mathcal{E}^{k}\right)$ be measurable. Then

$$
\begin{aligned}
M(\eta, B \times C):=\iint & \mathbf{1}\{x \in B\} \mathbf{1}\left\{\Theta\left(S^{\prime}\left(\left\{F_{1}-x, \ldots, F_{n}-x\right\}\right)\right) \in C\right\} \\
& \times \mathbf{1}\left\{\left\{F_{1}-x, \ldots, F_{n}-x\right\} \in H\right\} \\
& \times \mathbf{1}\left\{\eta_{F_{1}, \ldots, F_{n}}\left(S^{\prime}\left(\left\{F_{1}-x, \ldots, F_{n}-x\right\}\right)+x\right)=m\right\} \mathrm{d} x \\
& \times \eta^{(n)}\left(\mathrm{d}\left(F_{1}, \ldots, F_{n}\right)\right)
\end{aligned}
$$

is of the form (3.7). Indeed, we can take $S\left(F_{1}, \ldots, F_{n}, x\right):=S^{\prime}\left(\left\{F_{1}-x, \ldots, F_{n}-x\right\}\right)+x$, $R\left(F_{1}, \ldots, F_{n}, x\right):=\mathbf{1}\left\{\left\{F_{1}-x, \ldots, F_{n}-x\right\} \in H\right\}$, and $j=d$. Using stationarity and Fubini's theorem, we obtain

$$
\mathrm{E} M(X, B \times C)=\gamma_{m, n} \mathscr{H}^{d}(B) \mathbb{V}_{m, n}(C),
$$

where

$$
\gamma_{m, n}:=\mathrm{E} \int \mathbf{1}\left\{\left\{F_{1}, \ldots, F_{n}\right\} \in H\right\} \mathbf{1}\left\{X_{F_{1}, \ldots, F_{n}}^{!}\left(S^{\prime}\left(\left\{F_{1}, \ldots, F_{n}\right\}\right)\right)=m\right\} X^{(n)}\left(\mathrm{d}\left(F_{1}, \ldots, F_{n}\right)\right)
$$


and

$$
\begin{aligned}
\mathbb{V}_{m, n}:=\gamma_{m, n}^{-1} \mathrm{E} \int & \mathbf{1}\left\{\Theta\left(S^{\prime}\left(\left\{F_{1}, \ldots, F_{n}\right\}\right)\right) \in \cdot\right\} \mathbf{1}\left\{\left\{F_{1}, \ldots, F_{n}\right\} \in H\right\} \\
& \times \mathbf{1}\left\{X_{F_{1}, \ldots, F_{n}}\left(S^{\prime}\left(\left\{F_{1}, \ldots, F_{n}\right\}\right)\right)=m\right\} X^{(n)}\left(\mathrm{d}\left(F_{1}, \ldots, F_{n}\right)\right),
\end{aligned}
$$

where we assume that $\gamma_{m, n}>0$. We also assume that $S^{\prime}$ and $H$ are equivariant and, respectively, invariant under scaling. Then (3.5) holds and Theorem 3.1 implies that $\mathbb{V}_{m, n}=\Gamma(m+n, \gamma)$.

Under additional assumptions, the previous result can be expressed in a different way. So we assume that there is a point process $Y: N^{k} \rightarrow N^{k}$ which is almost surely uniquely determined by the conditions $Y \subset X, Y \in H$, and $(X \backslash Y)\left(S^{\prime}(Y)\right)=m$. We may call $Y$ a subprocess of $X$. From (4.1), it is obvious that

$$
\mathbb{V}_{m, n}=\mathrm{P}\left(\Theta\left(S^{\prime}(Y)\right) \in \cdot \mid Y\left(\mathcal{E}^{k}\right)=n\right),
$$

so that the conditional distribution of $\Theta\left(S^{\prime}(Y)\right)$ given $Y\left(\mathcal{E}^{k}\right)=n$ is $\Gamma(m+n, \gamma)$. We may also consider the scaled point process

$$
Y^{\prime}:=\left(\Theta\left(S^{\prime}(Y)\right)\right)^{-1 /(d-k)} Y
$$

where $a^{-1}:=0$ if $a=0$. Using the scaling properties of $S^{\prime}, H$, and $\Theta$, it is easy to see that, almost surely, $Y^{\prime}=G(X)$ for a scale-invariant measurable function $G: N^{k} \rightarrow N^{k}$. Using this $G$, we can define a stationary marked random measure $M_{G}$ by (3.9). The associated mark distribution equals $\mathrm{P}\left(\left(\Theta\left(S^{\prime}(Y)\right), Y^{\prime}\right) \in \cdot \mid Y\left(\mathcal{E}^{k}\right)=n\right)$. Therefore, we find from Theorem 3.2 that $\Theta\left(S^{\prime}(Y)\right)$ and $Y^{\prime}$ are conditionally independent given $Y\left(\varepsilon^{k}\right)=n$. In the $m=0$ case and the setting of Remark 3.4 these results are the content of Theorem 3 of [12].

\subsection{The measure of stopping sets}

In this subsection we consider the $n=0$ case, so that we can then work within the setting of Theorem 3.3. The stationary random measure $M^{\prime}$ defined by (3.18) is then given by

$$
M^{\prime}(\eta, B):=\int \mathbf{1}\{x \in B\} \mathbf{1}\left\{\eta\left(S^{\prime}(\eta-x)+x\right)=m\right\} R^{\prime}(\eta-x) \mathrm{d} x,
$$

where $S^{\prime}(\eta):=S(0, \eta)$ and $R^{\prime}(\eta):=R(0, \eta)$. From stationarity we obtain, for any $A \in \mathcal{N}^{k}$,

$$
\mathrm{E} \int_{B} \mathbf{1}\{X-x \in A\} M^{\prime}(X, \mathrm{~d} x)=\mathscr{H}^{d}(B) \mathrm{E} \mathbf{1}\{X \in A\} \mathbf{1}\left\{X\left(S^{\prime}\right)=m\right\} R^{\prime}
$$

Assuming that

$$
0<\gamma_{M^{\prime}}=\operatorname{E} \mathbf{1}\left\{X\left(S^{\prime}\right)=m\right\} R^{\prime}<\infty
$$

we derive that

$$
\mathrm{P}_{m}(\cdot):=\left(\mathrm{E} \mathbf{1}\left\{X\left(S^{\prime}\right)=m\right\} R^{\prime}\right)^{-1} \mathrm{E} \mathbf{1}\{X \in \cdot\} \mathbf{1}\left\{X\left(S^{\prime}\right)=m\right\} R^{\prime}
$$

is the Palm probability measure of $M^{\prime}$. The same arguments show that assumption (3.5) takes the form

$$
\mathrm{E} \mathbf{1}\left\{c X\left(S^{\prime}(c X)\right)=m\right\} R^{\prime}(c X)=\mathrm{E} \mathbf{1}\left\{X\left(S^{\prime}(X)\right)=m\right\} R^{\prime}(X), \quad c>0 .
$$

Under this assumption, Theorem 3.3 implies that $\Theta\left(S^{\prime}\right)$ is $\Gamma(m, \gamma)$-distributed under $\mathrm{P}_{m}$. Moreover, any scale-invariant random variable $G$ is $\mathrm{P}_{m}$-independent of $\Theta\left(S^{\prime}\right)$. In the $R^{\prime} \equiv 1$ case this is a special case of Theorem 2 of [17]. 


\section{Fundamental regions in Voronoi tessellation}

\subsection{Voronoi tessellations}

In this section we assume that $k=0$ and consider the Voronoi tessellation based on the Poison process $X$. Our aim is to apply the results from Section 3 to the fundamental region of the typical $j$-faces of the tessellation. We consider both the area-biased and the area-debiased cases.

We give here a few basic definitions and refer the reader to [11] and Section 10.2 of [14] for more details. Let $\eta \in N^{0}$. The Voronoi cell $C(\eta, x)$ of $x \in \eta$ consists of all points $y \in \mathbb{R}^{d}$ satisfying $|y-x| \leq \min \{|y-z|: z \in \eta\}$. Then $\delta_{d}(\eta):=\{C(\eta, x): x \in \eta\}$ is the Voronoi tessellation based on $\eta$. A cell is referred to as a $d$-face. Let $j \in\{0, \ldots, d-1\}$. A $j$-face (of $\left.\delta_{d}(\eta)\right)$ is a $j$-dimensional convex set which is the intersection of (at least) $d-j+1$ Voronoi cells $C\left(\eta, x_{1}\right), \ldots, C\left(\eta, x_{d-j+1}\right)$, where $x_{1}, \ldots, x_{d-j+1}$ are points of $\eta$. The system of all $j$-faces of the Voronoi tessellation $\varsigma_{d}(\eta)$ is denoted by $\varsigma_{j}(\eta)$.

\subsection{The fundamental region of the area-biased typical $\boldsymbol{j}$-face}

We fix $j \in\{0, \ldots, d\}$ and define

$$
M_{j}(\cdot):=\sum_{F \in \oiint_{j}(X)} \mathscr{H}^{j}(F \cap \cdot)
$$

as the random measure arising by restricting the $j$-dimensional Hausdorff measure to the $j$-faces of the Poisson-Voronoi tessellation $\delta_{d}(X)$. We will see below that this definition (after a possible modification on an invariant P-null set) yields a stationary random measure in the sense of Subsection 2.4. The intensity of $M_{j}$ is positive and finite; see, e.g. Theorem 10.2.4 of [14]. Under the Palm probability measure $\mathrm{P}_{M_{j}}$ (cf. (2.10)), the origin belongs almost surely to the relative interior of a $j$-face $L_{j}$, say, the area-biased typical $j$-face of $\S_{d}(X)$. Also, almost surely, there are $d-j+1$ points $\xi_{1}, \ldots, \xi_{d-j+1} \in X$ (the neighbors of $L_{j}$ ) such that

$$
L_{j}=C\left(X, \xi_{1}\right) \cap \cdots \cap C\left(X, \xi_{d-j+1}\right) .
$$

We call

$$
T_{j}:=\bigcup_{x \in \AA_{0}\left(L_{j}\right)} B\left(x,\left\|x-\xi_{1}\right\|\right)
$$

the fundamental region of $L_{j}$, where $\varsigma_{0}\left(L_{j}\right)$ is the set of vertices of $L_{j}$; see Figure 1 for the case in which $j=1$.

Theorem 5.1. Let $m \in \mathbb{N}_{0}$, and assume that $m=0$ when $j=0, m=2$ when $j=1$, and $m \geq j+1$ in all other cases. Consider the conditional probability measure $\mathrm{P}_{M_{j}}^{0}$ given that $X\left(T_{j}\right)=m+d-j+1$. Then the volume of the fundamental region $T_{j}$ has a $\Gamma(m+d-j+$ $j / d, \gamma)$-distribution.

This result is a consequence of Theorem 3.3. We defer the (technical) proof to Subsection 5.4.

Remark 5.1. In the situation of Theorem 5.1 assume that $X\left(T_{j}\right)=m+d-j+1$. Then the typical face $L_{j}$ has $m$ faces of dimension $j-1$.

Remark 5.2. The probability measure $\mathrm{P}_{M_{0}}^{0}$ describes $X$ as seen from the typical vertex $L_{0}=\{0\}$. Its fundamental region is the ball centered at 0 and having the $d+1$ nearest $X$-neighbors of 0 on its boundary. It is a classical fact (see [9]) that the volume of this ball has a $G(d, \gamma)$-distribution (see also [1] and Example 1.1). 


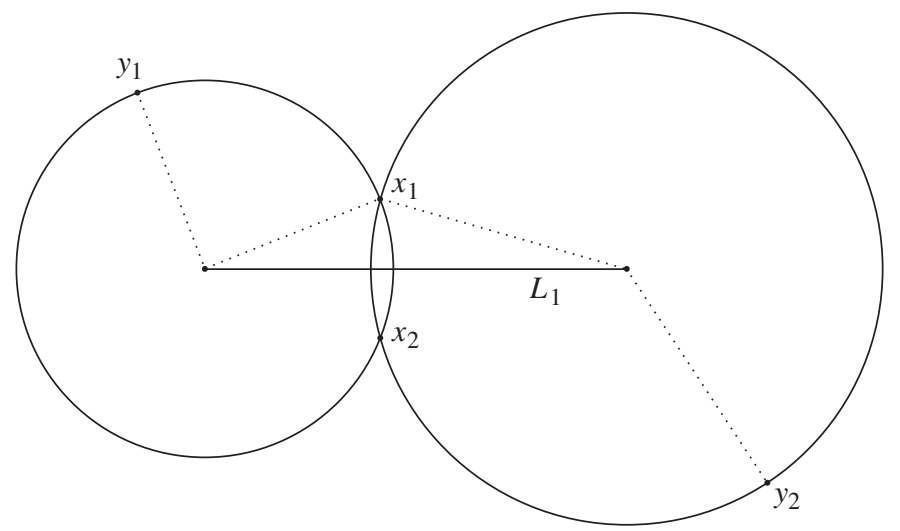

Figure 1: In this picture $L_{1}$ is an edge of the Voronoi tessellation generated by $X$. This edge is the intersection of Voronoi cells $C\left(X, x_{1}\right)$ and $C\left(X, x_{2}\right)$, where $x_{1}, x_{2} \in X$ are the neighbors of $L_{1}$. The fundamental region $T_{1}$ of $L_{1}$ is given by the union of the two balls. The vertices of $L_{1}$ are the intersection of $C\left(X, x_{1}\right), C\left(X, x_{2}\right)$, and $C\left(X, y_{1}\right)$, and $C\left(X, x_{1}\right), C\left(X, x_{2}\right)$, and $C\left(X, y_{2}\right)$, where $y_{1}, y_{2} \in X$.

Remark 5.3. In the case in which $j=d, M_{d}$ is the Lebesgue measure and $\mathrm{P}_{M_{d}}^{0}=\mathrm{P}$. Under $\mathrm{P}$, the face $L_{d}$ is the (stationary) 0 -cell, i.e. the cell containing the origin. The volume of its fundamental region $T_{d}$ is, by Theorem 5.1, conditionally $G(m+1, \gamma)$-distributed, given that $L_{d}$ has $m \geq d+1$ faces of dimension $d-1$. To our surprise, we were not able to find this result in the literature. (The case in which $d=1$ is clearly well known.)

Remark 5.4. The probability measure $\mathrm{P}_{M_{1}}^{0}$ describes $X$ as seen from the area-biased chosen typical edge $L_{1}$. The fundamental region $T_{1}$ is the union of two balls centered at the endpoints of $L_{0}$ and having their nearest $X$-neighbor on its boundary; see Figure 1. According to Theorem 5.1, the volume of $T_{1}^{*}$ is $\Gamma(d+1+1 / d, \gamma)$-distributed.

\subsection{The fundamental region of the area-debiased typical $\boldsymbol{j}$-face}

For any compact and nonempty set $C \subset \mathbb{R}^{d}$, we let $\pi(C)$ denote the center of the circumball of $C$. This center function has the equivariance property $\pi(C+x)=\pi(C)+x, x \in \mathbb{R}^{d}$. Fix $j \in\{0, \ldots, d\}$. If $C$ is a $j$-dimensional compact and nonempty convex set then $\pi(C)$ is in the relative interior of $C$. Define

$$
N_{j}:=\sum_{F \in S_{j}(X)} \delta_{\pi(F)}
$$

as the (simple) point process supported by the centers of $j$-faces of $\delta_{d}(X)$. This is clearly a stationary random measure. The intensity of $N_{j}$ is positive and finite; see, e.g. Theorem 10.2.4 of [14]. Under the Palm probability measure $\mathrm{P}_{N_{j}}$ (cf. (2.10)), the origin is almost surely a center of a $j$-face $C_{j}$, say, the area-debiased typical $j$-face of $\delta_{d}(X)$. Almost surely, with respect to $\mathrm{P}_{N_{j}}$, there are $d-j+1$ points $\xi_{1}^{\prime}, \ldots, \xi_{d-j+1}^{\prime} \in X$ (the neighbors of $C_{j}$ ) such that

$$
C_{j}=C\left(X, \xi_{1}^{\prime}\right) \cap \cdots \cap C\left(X, \xi_{d-j+1}^{\prime}\right) .
$$

We call

$$
T_{j}^{*}:=\bigcup_{x \in \oiint_{0}\left(C_{j}\right)} B\left(x,\left\|x-\xi_{1}^{\prime}\right\|\right)
$$

the fundamental region of $C_{j}$, where $\varsigma_{0}\left(C_{j}\right)$ is the set of vertices of $C_{j}$. 
Theorem 5.2. Let $m \in \mathbb{N}_{0}$, and assume that $m=0$ when $j=0, m=2$ when $j=1$, and $m \geq j+1$ in all other cases. Consider the conditional probability measure $\mathrm{P}_{N_{j}}^{0}$ given that $X\left(T_{j}^{*}\right)=m+d-j+1$. Then the volume of the fundamental region $T_{j}^{*}$ has $a \Gamma(m+d-j, \gamma)$ distribution.

This result is again a consequence of Theorem 3.3 and will be proved in Subsection 5.4. In the following we will comment on the cases in which $j=d$ and $j=1$. The classical special case, $j=0$, has already been discussed in Remark 5.2. The remaining special cases seem to be new.

Remark 5.5. Under $\mathrm{P}_{d}$, the face $C_{d}$ is the typical cell of $\delta_{d}(X)$. The volume of its fundamental region $S_{d}$ is conditionally $G(m, \gamma)$-distributed, given that $C_{d}$ has $m \geq d+1$ faces of dimension $(d-1)$. This special case of Theorem 5.2 is well known; see [10] and [12]. Note the difference in the shape parameter when compared with the stationary 0-cell alluded to in Remark 5.3. The reader is advised to consider the case in which $d=1$ to explain this phenomenon. In this case the fundamental region of a randomly picked cell has a $G(2, \gamma)$-distributed length, while a length-biased chosen cell has a fundamental region with a $G(3, \gamma)$-distributed length.

Remark 5.6. The probability measure $\mathrm{P}_{N_{1}}^{0}$ describes $X$ as seen from the typical edge $C_{1}$ of $\wp_{d}(X)$. Similarly as in the area-biased case (see Remark 5.4 and Figure 1), the fundamental region $T_{1}^{*}$ is the union of two balls centered at the endpoints of $C_{0}$. Using different methods, it has been shown in [1] (and in [3] for $d=2$ ) that the volume of the fundamental region of $C_{1}$ (see Figure 1) has a $G(d+1, \gamma)$-distribution. This is in accordance with Theorem 5.2.

\subsection{Proofs of Theorems 5.1 and 5.2}

Let $j \in\{0, \ldots, d\}$, let $x_{1}, \ldots, x_{d-j+1} \in \mathbb{R}^{d}$, and, for $j<d$, assume that these points are in general position, i.e. not contained in some affine space of dimension $d-j-1$. For such points, we define

$$
Z_{j}\left(x_{1}, \ldots, x_{d-j+1}\right):=\left\{y \in \mathbb{R}^{d}:\left|y-x_{j}\right|=\left|y-x_{1}\right|, j=2, \ldots, d-j+1\right\} .
$$

If the closed set

$$
L_{j}\left(x_{1}, \ldots, x_{d-j+1}, \eta\right):=\left\{x \in Z_{j}\left(x_{1}, \ldots, x_{d-j+1}\right): \eta \cap B^{0}\left(x,\left|x-x_{1}\right|\right)=\varnothing\right\}
$$

has nonempty relative interior, then $L_{j}\left(x_{1}, \ldots, x_{d-j+1}, \eta\right)$ is a $j$-face of the Voronoi tessellation based on $\eta \cup\left\{x_{1}, \ldots, x_{d-j+1}\right\}$. If $L_{j}\left(x_{1}, \ldots, x_{d-j+1}, \eta\right)$ is compact and nonempty, we define

$$
S_{j}\left(x_{1}, \ldots, x_{d-j+1}, \eta\right):=\cup B\left(y,\left|y-x_{1}\right|\right),
$$

where the union is over all vertices $y$ of $L_{j}\left(x_{1}, \ldots, x_{d-j+1}, \eta\right)$; see Figure 1. Otherwise, we let $S_{j}\left(x_{1}, \ldots, x_{d-j+1}, \eta\right):=\mathbb{R}^{d}$. If $x_{1}, \ldots, x_{d-j+1} \in \mathbb{R}^{d}$ are not in general position, we set

$$
L_{j}\left(x_{1}, \ldots, x_{d-j+1}, \eta\right):=Z_{j}\left(x_{1}, \ldots, x_{d-j+1}\right):=\varnothing, \quad S_{j}\left(x_{1}, \ldots, x_{d-j+1}, \eta\right):=\mathbb{R}^{d} .
$$

It is quite standard to show that $Z_{j}, L_{j}$, and $S_{j}$ are measurable mappings. Moreover, these mappings are all equivariant under translations and scaling. The proof of the following geometrically obvious properties is also left to the reader. For any $x_{1}, \ldots, x_{d-j+1} \in \mathbb{R}^{d}$ and $\eta, \psi \in N^{0}$ with $\psi \subset S_{j}\left(x_{1}, \ldots, x_{d-j+1}, \eta\right)^{\mathrm{c}}$, we have

$$
\begin{aligned}
& L_{j}\left(x_{1}, \ldots, x_{d-j+1}, \eta\right)=L_{j}\left(x_{1}, \ldots, x_{d-j+1}, \eta \cap S_{j}\left(x_{1}, \ldots, x_{d-j+1}, \eta\right) \cup \psi\right), \\
& S_{j}\left(x_{1}, \ldots, x_{d-j+1}, \eta\right)=S_{j}\left(x_{1}, \ldots, x_{d-j+1}, \eta \cap S_{j}\left(x_{1}, \ldots, x_{d-j+1}, \eta\right) \cup \psi\right), \\
& x \in L_{j}\left(x_{1}, \ldots, x_{d-j+1}, \eta\right) \Longrightarrow B\left(x,\left|x-x_{1}\right|\right) \subset S_{j}\left(x_{1}, \ldots, x_{d-j+1}, \eta\right) .
\end{aligned}
$$


Equation (5.4) is the reason for calling $S_{j}\left(x_{1}, \ldots, x_{d-j+1}, \eta\right)$ the fundamental region of the face $L_{j}$ : changing the underlying configuration outside $S_{j}$ has no influence on the shape of $L_{j}$.

Lemma 5.1. For any $x_{1}, \ldots, x_{d-j+1} \in \mathbb{R}^{d}$, the mapping $S_{j}\left(x_{1}, \ldots, x_{d-j+1}, \cdot\right): N^{0} \rightarrow$ $\mathcal{F}$ is a stopping set and $L_{j}\left(x_{1}, \ldots, x_{d-j+1}, \cdot\right): N^{0} \rightarrow \mathcal{F}$ is measurable with respect to $\mathcal{N}_{S_{j}\left(x_{1}, \ldots, x_{d-j+1}, \cdot\right)}$.

Proof. The second assertion follows by (5.4) and Lemma A.2(i). To prove the first assertion, we omit $x_{1}, \ldots, x_{d-j+1}$ in the argument of $S_{j}$. Let $\eta, \psi \in N^{0}$ with $\psi=\eta \cap S_{j}(\psi)$. Using $\psi \subset S_{j}(\psi), \eta=\psi \cup\left(\eta \cap S_{j}(\psi)^{\mathrm{c}}\right)$, and (5.5), we obtain $S_{j}(\psi)=S_{j}(\eta)$. Equation (5.5) implies that $S_{j}(\eta)=S_{j}\left(\eta \cap S_{j}(\eta)\right)$. By Proposition A.1, $S_{j}$ is a stopping set.

Lemma 5.2. For any $x_{1}, \ldots, x_{d-j+1}, x \in \mathbb{R}^{d}$, the mapping

$$
R\left(x_{1}, \ldots, x_{d-j+1}, x, \cdot\right): \eta \mapsto \mathbf{1}\left\{\eta\left(B^{0}\left(x,\left|x-x_{1}\right|\right)\right)=0, x \in Z_{j}\left(x_{1}, \ldots, x_{d-j+1}\right)\right\}
$$

is invariant under translations and scaling and measurable with respect to $\mathcal{N}_{S_{j}\left(x_{1}, \ldots, x_{d-j+1}, \cdot\right)}$.

Proof. The invariance property is clear. To prove the stated property of measurablity, we fix $x, x_{1}, \ldots, x_{d-j+1} \in \mathbb{R}^{d}$ and omit them in the argument of $R, S_{j}$, and $L_{j}$. Obviously, $R(\eta) \leq R\left(\eta \cap S_{j}(\eta)\right)$. Assume that $R\left(\eta \cap S_{j}(\eta)\right)=1$. Then we have, by (5.4), $x \in$ $L_{j}\left(\eta \cap S_{j}(\eta)\right)=L_{j}(\eta)$, and, by (5.6),

$$
\varnothing=\eta \cap S_{j}(\eta) \cap B^{0}\left(x,\left|x-x_{1}\right|\right)=\eta \cap B^{0}\left(x,\left|x-x_{1}\right|\right) .
$$

Hence, $R(\eta)=1$. This shows that $R(\eta)=R\left(\eta \cap S_{j}(\eta)\right)$, so that the assertion follows from Lemma A.2(i).

With $m \geq 0$ and $j \geq 0$ fixed as in the previous sections we define stationary measures $M_{j, m}$ and $M_{j}$ by

$$
\begin{aligned}
M_{j, m}(\eta, \cdot):=\iint & \mathbf{1}\left\{x \in Z_{j}\left(x_{1}, \ldots, x_{d-j+1}\right) \cap \cdot\right\} \mathbf{1}\left\{\eta_{x_{1}, \ldots, x_{d-j+1}}^{!}\left(B^{0}\left(x,\left|x-x_{1}\right|\right)\right)=0\right\} \\
& \times \mathbf{1}\left\{\eta_{x_{1}, \ldots, x_{d-j+1}}\left(S_{j}\left(x_{1}, \ldots, x_{d-j+1}, \eta_{x_{1}, \ldots, x_{d-j+1}}\right)\right)=m\right\} \\
& \times \mathcal{H}^{j}(\mathrm{~d} x) \eta^{(d-j+1)}\left(\mathrm{d}\left(x_{1}, \ldots, x_{d-j+1}\right)\right)
\end{aligned}
$$

and

$$
\begin{aligned}
M_{j}(\eta, \cdot):=\iint & \mathbf{1}\left\{x \in Z_{j}\left(x_{1}, \ldots, x_{d-j+1}\right) \cap \cdot\right\} \mathbf{1}\left\{\eta_{x_{1}, \ldots, x_{d-j+1}}^{!}\left(B^{0}\left(x,\left|x-x_{1}\right|\right)\right)=0\right\} \\
& \times \mathcal{H}^{j}(\mathrm{~d} x) \eta^{(d-j+1)}\left(\mathrm{d}\left(x_{1}, \ldots, x_{d-j+1}\right)\right) .
\end{aligned}
$$

Almost surely, the random measure $M_{j, m}(X, \cdot)$ is the restriction of $\mathscr{H}^{j}$ onto the union of all $j$-faces of $\delta_{d}(\eta)$ having exactly $m(j-1)$-faces on their boundary. In particular, $M_{j}(X, \cdot)$ coincides almost surely with the random measure defined by (5.1). The following lemma justifies our terminology.

Lemma 5.3. For any $\eta \in N^{0}$, the measure $M_{j}(\eta, \cdot)$ is locally finite.

Proof. Let $\eta \in N^{0}$. Note that, for all $x_{1}, \ldots, x_{d-j+1} \in \mathbb{R}^{d}$, we have

$$
\mathscr{H}^{j}\left(Z_{j}\left(x_{1}, \ldots, x_{d-j+1}\right) \cap B^{d}\right) \leq \kappa_{j},
$$


where $\kappa_{j}$ denotes the volume of the unit ball in $\mathbb{R}^{j}$. Fix $y \in \eta$, and let $x_{1}, \ldots, x_{d-j+1} \in \eta$ be in general position. If $B^{0}\left(x,\left|x-x_{1}\right|\right) \cap \eta=\varnothing$ for some $x \in B^{d} \cap Z_{j}\left(x_{1}, \ldots, x_{d-j+1}\right)$ then

$$
B^{0}\left(x,\left|x-x_{i}\right|\right) \cap \eta=\varnothing, \quad i=1, \ldots, d-j+1 .
$$

In particular, we have $y \notin B^{0}\left(x,\left|x-x_{i}\right|\right)$ for $i=1, \ldots, d-j+1$. Hence,

$$
|y|+1 \geq|y-x| \geq\left|x_{i}-x\right| \geq\left|x_{i}\right|-1, \quad i=1, \ldots, d-j+1,
$$

and we conclude that $\left|x_{i}\right| \leq|y|+2$ for $i=1, \ldots, d-j+1$. We obtain

$$
M_{j}\left(\eta, B^{d}\right) \leq \kappa_{j} \eta(B(0,|y|+2))^{d-j+1}<\infty .
$$

The assertion follows by $M_{j}(\eta, B+x)=M_{j}(\eta-x, B)$ for $x \in \mathbb{R}^{d}$ and $B \in \mathscr{B}^{d}$.

The intensity of $M_{j, m}$ is positive if and only if one of the following cases hold: $j=0$ and $m=0, j=1$ and $m=2$, or $j \in\{2, \ldots, d\}$ and $m \geq j+1$. In the remainder of this subsection we will only consider these cases.

Let $\eta \in N^{0}$ and $x \in \mathbb{R}^{d}$. If 0 is in the relative interior of some $j$-face $L$ of $\delta_{d}(\eta)$ and if $L$ is the intersection of exactly $d-j+1$ Voronoi cells $C\left(\eta, x_{1}\right), \ldots, C\left(\eta, x_{d-j+1}\right)$, where $x_{1}, \ldots, x_{d-j+1}$ are lexicographically ordered points of $\eta$, then we define $L_{j}(\eta):=L$ and

$$
g_{1}(\eta):=x_{1}, \ldots, g_{d-j+1}(\eta):=x_{d-j+1} .
$$

Otherwise, we set $L_{j}(\eta):=\{0\}$ and $g_{i}(\eta):=0, i=1, \ldots, d-j+1$. We define

$$
T_{j}(\eta):=S_{j}\left(g_{1}(\eta), \ldots, g_{d-j+1}(\eta), \eta \backslash\left\{g_{1}(\eta), \ldots, g_{d-j+1}(\eta)\right\}\right) .
$$

Obviously,

$$
T_{j}(X)=T_{j} \quad \mathrm{P} \text {-almost surely, }
$$

where the fundamental region $T_{j}$ has been defined in Subsection 5.2. The refined Campbell theorem, (2.10), easily implies that $\gamma_{M_{j, m}}=\gamma_{M_{j}} \mathrm{P}_{M_{j}}^{0}\left(X\left(T_{j}\right)=m+d-j+1\right)$ and

$$
\mathrm{P}_{M_{j, m}}^{0}=\mathrm{P}_{M_{j}}^{0}\left(\cdot \mid X\left(T_{j}\right)=m+d-j+1\right) .
$$

Using Lemmas 5.1 and 5.2, we may now apply Theorem 3.3 with $n=d-j+1$ to show that the distribution of the volume of $T_{j}$ under $\mathrm{P}_{M_{j, m}}^{0}$ is $\Gamma(d+m-j+j / d, \gamma)$. In view of (5.7) and (5.8), this is Theorem 5.1.

We next prove Theorem 5.2. For $x_{1}, \ldots, x_{d-j+1} \in \mathbb{R}^{d}$ in general position, we denote by $z\left(x_{1}, \ldots, x_{d-j+1}\right)$ the center of the uniquely determined $(d-j)$-dimensional ball having $x_{1}, \ldots, x_{d-j+1}$ on its boundary. If $x_{1}, \ldots, x_{d-j+1} \in \mathbb{R}^{d}$ are not in general position, we set $z\left(x_{1}, \ldots, x_{d-j+1}\right):=\infty$, where $\infty$ is some point outside $\mathbb{R}^{d}$. We define a stationary measure $N_{j, m}$ by

$$
\begin{aligned}
N_{j, m}(\eta, \cdot):=\int & \mathbf{1}\left\{L_{j}\left(x_{1}, \ldots, x_{d-j+1}, \eta_{x_{1}, \ldots, x_{d-j+1}}\right) \neq \varnothing\right\} \mathbf{1}\left\{z\left(x_{1}, \ldots, x_{d-j+1}\right) \in \cdot\right\} \\
& \times \mathbf{1}\left\{\eta_{x_{1}, \ldots, x_{d-j+1}}\left(S_{j}\left(x_{1}, \ldots, x_{d-j+1}, \eta_{x_{1}, \ldots, x_{d-j+1}}\right)\right)=m\right\} \\
& \times \eta^{(d-j+1)}\left(\mathrm{d}\left(x_{1}, \ldots, x_{d-j+1}\right)\right),
\end{aligned}
$$


if

$$
\begin{aligned}
N_{j}^{\prime}(\eta, \cdot):=\int & \mathbf{1}\left\{L_{j}\left(x_{1}, \ldots, x_{d-j+1}, \eta_{x_{1}, \ldots, x_{d-j+1}}^{!}\right) \neq \varnothing\right\} \mathbf{1}\left\{z\left(x_{1}, \ldots, x_{d-j+1}\right) \in \cdot\right\} \\
& \times \eta^{(d-j+1)}\left(\mathrm{d}\left(x_{1}, \ldots, x_{d-j+1}\right)\right)
\end{aligned}
$$

is a locally finite measure. Otherwise, we let $N_{j, m}(\eta, \cdot)$ and $N_{j}^{\prime}(\eta, \cdot)$ be the zero measure. The right-hand side of (5.9) is in general neither simple (see Subsection 2.4) nor locally finite. However, the right-hand side of (5.10) is, for P-a.e., $\eta$ simple as well as locally finite. Because of the choice of $m$ and $j$ made above, the intensity of $N_{j, m}$ is positive.

If $N_{j}^{\prime}(\eta, \cdot)$ is simple and has the origin in its support, then there exist a uniquely determined $j$-face $C_{j}^{\prime}(\eta)$ of $s_{d}(\eta)$ and lexicographically ordered points $x_{1}, \ldots, x_{d-j+1} \in \eta$ with $C_{j}^{\prime}(\eta)=L_{j}\left(x_{1}, \ldots, x_{d-j+1}, \eta\right)$ and $z\left(x_{1}, \ldots, x_{d-j+1}\right)=0$. If, in addition, the points $x_{1}, \ldots, x_{d-j+1} \in \eta$ are uniquely determined by $C_{j}^{\prime}(\eta)$, then we define

$$
h_{1}(\eta):=x_{1}, \ldots, h_{d-j+1}(\eta):=x_{d-j+1} .
$$

Otherwise, we set $h_{i}(\eta):=0, i=1, \ldots, d-j+1$. We define

$$
T_{j}^{\prime}(\eta):=S_{j}\left(h_{1}(\eta), \ldots, h_{d-j+1}(\eta), \eta \backslash\left\{h_{1}(\eta), \ldots, h_{d-j+1}(\eta)\right\}\right)
$$

and

$$
T_{j}^{\prime}:=T_{j}^{\prime}(X)
$$

As at (5.8), we have $\gamma_{N_{j, m}}=\gamma_{N_{j}^{\prime}} \mathrm{P}_{N_{j}^{\prime}}^{0}\left(X\left(T_{j}^{\prime}\right)=m+d-j+1\right)$ and

$$
\mathrm{P}_{N_{j, m}}^{0}=\mathrm{P}_{N_{j}^{\prime}}^{0}\left(\cdot \mid X\left(T_{j}^{\prime}\right)=m+d-j+1\right) .
$$

Using Lemma 5.1, we may now apply Theorem 3.3 with $n=d-j+1$ and $j$ replaced with 0 to obtain the following result.

Proposition 5.1. The distribution of the volume of $T_{j}^{\prime}$ under $\mathrm{P}_{N_{j, m}}^{0}$ is $\Gamma(d+m-j, \gamma)$.

To derive Theorem 5.2 from Proposition 5.1, we need a relationship between the Palm probability measures $\mathrm{P}_{N_{j}^{\prime}}^{0}$ and $\mathrm{P}_{N_{j}}^{0}$. The random measures $N_{j}^{\prime}$ and $N_{j}$ are different. However, we have the intuitively plausible equations $\gamma_{N_{j}}=\gamma_{N_{j}^{\prime}}$ and

$$
\mathrm{P}_{N_{j}}^{0}(\cdot)=\mathrm{P}_{N_{j}^{\prime}}^{0}\left(X-\pi\left(C_{j}^{\prime}\right) \in \cdot\right),
$$

where $C_{j}^{\prime}:=C_{j}^{\prime}(X)$. A formal proof can, e.g. be based on Theorem 4.1 of [5]. Clearly, we can write the fundamental region defined by (5.3) in the form $S_{j}=S_{j}(X)$ for a well-defined measurable mapping $S_{j}: N^{0} \rightarrow \mathcal{F}$. With this notation we have

$$
S_{j}\left(X-\pi\left(C_{j}^{\prime}\right)\right)=S_{j}^{\prime}-\pi\left(C_{j}^{\prime}\right) \quad \mathrm{P}_{N_{j}^{\prime}}^{0} \text {-almost surely. }
$$

Since $\Theta$ is translation invariant, it follows that

$$
\mathrm{P}_{N_{j}}^{0}\left(\Theta\left(T_{j}^{*}\right) \in \cdot, X\left(T_{j}^{*}\right)=m+d-j+1\right)=\mathrm{P}_{N_{j}^{\prime}}^{0}\left(\Theta\left(T_{j}^{\prime}\right) \in \cdot X\left(T_{j}^{\prime}\right)=m+d-j+1\right) .
$$

Hence, Theorem 5.2 follows from Proposition 5.1 and (5.11). 


\section{Typical faces of a Poisson hyperplane tessellation}

\subsection{Hyperplane tessellations}

In this section we assume that $k=d-1$ and consider the hyperplane tessellation based on the Poison process $X$. We assume that $X$ is nondegenerate in the sense of Section 10.3 of [14]; see also Example 1.3. We will use our results from Section 3 to show that the generalized integralgeometric contents of these faces (see (2.4)) are conditionally gamma distributed. Again, we consider the area-biased and the area-debiased cases.

We now give some basic definitions. More details can be found in Section 10.3 of [14]. Let $\eta \in N^{d-1}$. The connected components of the complement of the union $\bigcup_{F \in \eta} F$ is made up of open polyhedral sets. The closures of these cells form the hyperplane tessellation $\wp_{d}(\eta)$. Let $j \in\{0, \ldots, d-1\}$. A $j$-face ( of $\delta_{d}(\eta)$ ) is a $j$-dimensional convex set which is the intersection of one of the cells in $\delta_{d}(\eta)$ with a supporting hyperplane of this cell. The system of all $j$-faces of the hyperplane tessellation $\delta_{d}(\eta)$ is denoted by $\varsigma_{j}(\eta)$.

\subsection{Area-biased and area-debiased typical faces}

We fix $j \in\{1, \ldots, d\}$ and define the random measure $M_{j}$ by (5.1). Again, we will see below that this definition yields a stationary random measure in the sense of Subsection 2.4 (after a possible modification on an invariant P-null set). The intensity of $M_{j}$ is positive and finite; see, e.g. Theorem 10.3.3 of [14]. Under the Palm probability measure $\mathrm{P}_{M_{j}}^{0}$, the origin belongs almost surely to the relative interior of a $j$-face $L_{j}$, say. This is the area-biased typical $j$-face of $\delta_{d}(X)$. Recall the notation (2.1) and (2.6). In particular, $X\left(\varepsilon_{L_{j}}^{d-1}\right)$ is the number of hyperplanes in $X$ intersecting $L_{j}$.

Theorem 6.1. Let $m \in \mathbb{N}$, and assume that $m=2$ when $j=1$ and $m \geq j+1$ in all other cases. Consider the conditional probability measure $\mathrm{P}_{M_{j}}^{0}$ under the condition that $X\left(\varepsilon_{L_{j}}^{d-1}\right)=$ $m+d-j$. Then $\Theta^{*}\left(L_{j}\right)$ has a $\Gamma(m, \gamma)$-distribution.

Remark 6.1. Assume that $X\left(\varepsilon_{L_{j}}^{d-1}\right)=m+d-j$. Then the face $L_{j}$ has $m$ faces of dimension $j-1$.

Next we define a random measure $N_{j}$ by (5.2). Formally, we have to restrict the sum in (5.10) to all bounded $j$-faces. However, by Theorem 10.3.2 of [14], all cells and, hence, all $j$-faces are P-almost surely bounded. Therefore, this definition yields a stationary random measure, at least after a possible modification on an invariant P-null set. The intensity of $N_{j}$ is positive and finite; see, e.g. Theorem 10.3.3 of [14]. Under the Palm probability measure $\mathrm{P}_{N_{j}}$, the origin is almost surely a center of a $j$-face $C_{j}$, say. This is the area-debiased typical $j$-face of $\S_{d}(X)$.

Theorem 6.2. Let $m \in \mathbb{N}$, and assume that $m=2$ when $j=1$ and $m \geq j+1$ in all other cases. Consider the conditional probability measure $\mathrm{P}_{N_{j}}^{0}$ under the condition that $X\left(\varepsilon_{C_{j}}^{d-1}\right)=$ $m+d-j$. Then $\Theta^{*}\left(C_{j}\right)$ has a $\Gamma(m-j, \gamma)$-distribution.

Remark 6.2. In the isotropic case, $\Theta^{*}\left(L_{j}\right)$ and $\Theta^{*}\left(C_{j}\right)$ are respectively proportional to the mean breadth of $L_{j}$ and $C_{j}$. Then Theorems 6.1 and 6.2 provide very explicit information about the distribution of a typical face. The special case $j=1$ has already been treated in Examples 1.4 and 1.5.

Remark 6.3. The isotropic case $d=j=2$ of Theorem 6.2 can be found in [6, p. 185]. The $j=d$ case of Theorems 6.1 and 6.2 can be found in Example (v) of [12, Section 5]; see also [6, p. 177] for Theorem 6.1. In [12], Theorem 6.1 is proved with the help of subprocesses, as 
discussed in Subsection 4.2, while Theorem 6.2 is proved using Miles's original version of the complementary theorem (see Subsection 4.1). (In the second case [12] provides no information on how to actually choose the center function.)

\subsection{Proofs of Theorems 6.1 and 6.2}

A locally finite system of hyperplanes is said to be in general position if, for any $k \in$ $\{0, \ldots, d-1\}$, any $F \in \mathcal{E}^{k}$ is contained in at most $d-k$ hyperplanes of the system.

Proof of Theorem 6.1. Let $F_{1}, \ldots, F_{d-j+m} \in \mathcal{E}^{d-1}$, and consider the union of the sets $F_{1} \cap \cdots \cap F_{d-j} \cap F_{i}$ for $i=d-j+1, \ldots, d-j+m$. The closures of the connected components of the complement of this union in $F_{1} \cap \cdots \cap F_{d-j}$ are denoted by $s_{j}\left(F_{1}, \ldots, F_{d-j+m}\right)$. (For $j=d$, we define $F_{1} \cap \cdots \cap F_{d-j}:=\mathbb{R}^{d}$.) Let $\S_{j, m}\left(F_{1}, \ldots, F_{d-j+m}\right)$ denote the (finite) set of $j$-dimensional polytopes with $m(j-1)$-dimensional faces. Let

$$
\mu\left(F_{1}, \ldots, F_{d-j+m}, \cdot\right):=\sum_{F \in \mathcal{S}_{j, m}\left(F_{1}, \ldots, F_{d-j+m}\right)} \mathcal{H}^{j}(F \cap \cdot) .
$$

This measure is 0 if the dimension $F_{1} \cap \cdots \cap F_{d-j}$ is greater than $j$ or if $s_{j, m}\left(F_{1}, \ldots, F_{d-j+m}\right)$ is empty. For $x \in \mathbb{R}^{d}$, we let $C_{j}\left(F_{1}, \ldots, F_{d-j+m}, x\right):=C$ if $C \in \delta_{j, m}\left(F_{1}, \ldots, F_{d-j+m}\right)$ and $x$ is in the relative interior of $C$. In all other cases we define $C_{j}\left(F_{1}, \ldots, F_{d-j+m}, x\right):=\varnothing$. Define

$$
\begin{aligned}
M_{j, m}(\eta, \cdot):=\iint & \mathbf{1}\{x \in \cdot\} \mathbf{1}\left\{\left(\eta_{F_{1}, \ldots, F_{d-j+m}}^{!}\left(\mathcal{E}_{C_{j}\left(F_{1}, \ldots, F_{d-j+m}, x\right)}^{d-1}\right)=0\right\}\right. \\
& \times \mu\left(F_{1}, \ldots, F_{d-j+m}, \mathrm{~d} x\right) \eta^{(d-j+m)}\left(\mathrm{d}\left(F_{1}, \ldots, F_{d-j}\right)\right), \quad \eta \in N^{d-1} .
\end{aligned}
$$

Next we show that

$$
\sum_{F \in \delta_{j, m}(X)} \mathscr{H}^{j}(F \cap \cdot)=M_{j, m}(X, \cdot) \quad \text { P -almost surely, }
$$

where $\S_{j, m}(X)$ is the system of all $j$-faces in $\S_{j}(X)$ with $m$ faces of dimension $j-1$. Let $C \subset \mathbb{R}^{d}$ be a $j$-dimensional bounded polytope with $m(j-1)$-dimensional faces. Assume that there are pairwise different $F_{1}, \ldots, F_{d-j+m} \in X$ in general position such that

(i) $C \in \delta_{j, m}\left(F_{1}, \ldots, F_{d-j+m}\right)$,

(ii) $X_{F_{1}, \ldots, F_{d-j+m}}^{!}\left(\varepsilon_{C}^{d-1}\right)=0$.

Then $C \in \wp_{j}(X)$. Assume, conversely, that the latter is true. By Theorem 10.3.2 of [14], there exist almost surely uniquely determined (up to the order) $F_{1}, \ldots, F_{d-j+m} \in X$ in general position satisfying (i) and (ii). Hence, (6.2) holds.

By Theorem 10.3.3 of [14], the intensity $\gamma_{M_{j, m}}$ of $M_{j, m}$ is finite. In the case in which $j=1$ it is positive only when $m=2$. Equation (6.2) easily implies that

$$
\mathrm{P}_{M_{j, m}}^{0}=\mathrm{P}_{M_{j}}^{0}\left(\cdot \mid X\left(\varepsilon_{L_{j}}^{d-1}\right)=m+d-j\right)
$$

holds similarly as at (5.8).

To obtain Theorem 6.1, we construct an equivariant measurable mapping $L_{j}^{*}$ defined on $\varepsilon^{d-1}$, taking values in the space of convex subsets of $\mathbb{R}^{d}$, and having the property that $L_{j}=L_{j}^{*}(X)$ holds $\mathrm{P}_{M_{j, m}}^{0}$-almost surely. In fact, we can construct mappings

$$
g_{i}: N^{k} \rightarrow \mathcal{E}^{d-1}, \quad i \in\{1, \ldots, d-j+m\},
$$


such that, for all $\eta \in N^{k}$ and all pairwise different $F_{1}, \ldots, F_{n} \in \eta$,

$$
\left\{g_{1}(\eta-x)+x, \ldots, g_{d-j+m}(\eta-x)+x\right\}=\left\{F_{1}, \ldots, F_{d-j+m}\right\}
$$

for $\mu\left(F_{1}, \ldots, F_{d-j+m}, \cdot\right)$-a.e. $x \in \mathbb{R}^{d}$ with $\eta_{F_{1}, \ldots, F_{d-j+m}}^{!}\left(\mathcal{E}_{C_{j}\left(F_{1}, \ldots, F_{d-j+m}, x\right)}^{d-1}\right)=0$. (The details are left to the reader.) We can then take $L_{j}^{*}(\eta):=C_{j}\left(g_{1}(\eta), \ldots, g_{d-j+m}(\eta)\right)$. It is now easy to verify that the assumptions of Theorem 3.3 are all satisfied (with $n:=d-j+m$ and $m:=0$ ), to conclude that the distribution of $L_{j}^{*}$ under $\mathrm{P}_{M_{j, m}}^{0}$ is $\Gamma(m, \gamma)$. In view of (6.3), this implies Theorem 6.1.

Proof of Theorem 6.2. For $F_{1}, \ldots, F_{d-j+m} \in \mathcal{E}^{d-1}$, we define $\delta_{j, m}\left(F_{1}, \ldots, F_{d-j+m}\right)$ as in the proof of Theorem 6.1. Instead of (6.1) we now define

$$
\mu\left(F_{1}, \ldots, F_{d-j+m}, \cdot\right):=\sum_{F \in \delta_{j, m}\left(F_{1}, \ldots, F_{d-j+m}\right)} \delta_{\pi(F)}(\cdot) .
$$

For $x \in \mathbb{R}^{d}$, we define $C_{j}\left(F_{1}, \ldots, F_{d-j+m}, x\right)$ as in the proof of Theorem 6.1. Let

$$
\begin{aligned}
\tilde{N}_{j, m}(\eta, \cdot):=\iint & \mathbf{1}\{x \in \cdot\} \mathbf{1}\left\{\eta_{F_{1}, \ldots, F_{d-j+m}}^{!}\left(\mathcal{E}_{C_{j}\left(F_{1}, \ldots, F_{d-j+m}, x\right)}^{d-1}\right)=0\right\} \mu\left(F_{1}, \ldots, F_{d-j+m}, \mathrm{~d} x\right) \\
& \times \eta^{(d-j+m)}\left(\mathrm{d}\left(F_{1}, \ldots, F_{d-j+m}\right)\right), \quad \eta \in N^{d-1} .
\end{aligned}
$$

The arguments used to establish (6.2) also show that $\sum_{F \in \delta_{j, m}(X)} \delta_{\pi(F)=\tilde{N}_{j, m}(X, \cdot)}$ P-almost surely. Therefore, it is easy to see that $\mathrm{P}_{\tilde{N}_{j, m}}^{0}=\mathrm{P}_{N_{j}}^{0}\left(\cdot \mid X\left(\mathcal{E}_{C_{j}}^{d-1}\right)=m+d-j\right)$. The assertion now follows similarly as in the proof of Theorem 6.1.

Remark 6.4. Let $m \geq d+1$, and let $F_{1}, \ldots, F_{m} \in \mathcal{E}^{d-1}$ be in general position. At first glance one might think that the tessellation $\wp_{d}\left(F_{1}, \ldots, F_{m}\right)$ (introduced in the proof of Theorem 6.1) contains at most one bounded cell with $m$ faces. Jürgen Kampf pointed out to us that this is true in the $d=2$ case but not true in higher dimensions. This explains why we have to work with the sum of possibly several Dirac measures in (6.4). Apparently, it is not enough to work with just one centroid associated with $F_{1}, \ldots, F_{m}$.

\section{Appendix A. Stopping sets}

In this appendix we present some basic results on stopping sets in a general setting. Let $\boldsymbol{Y}$ be a locally compact second countable Hausdorff space. The system of all closed subsets of $\boldsymbol{Y}$ is denoted by $\mathcal{F}(\boldsymbol{Y})$. On $\mathcal{F}(\boldsymbol{Y})$ we consider the smallest $\sigma$-field containing $\mathcal{F}_{K}:=\{F \in$ $\mathcal{F}(\boldsymbol{Y}): F \cap K \neq \varnothing\}$ for all compact $K \subset \boldsymbol{Y}$.

Let $(\boldsymbol{W}, \mathcal{W})$ be a measurable space, and let $\pi_{K}, K \in \mathcal{F}(\boldsymbol{Y})$, be a family of measurable mappings $\pi_{K}: W \rightarrow W$ with

$$
\pi_{K_{1}} \circ \pi_{K_{2}}=\pi_{K_{1}}, \quad K_{1} \subset K_{2}, K_{1}, K_{2} \in \mathcal{F}(\boldsymbol{Y}) .
$$

Two examples of such mappings are given in (A.5) and (A.7), below. The $\sigma$-field generated by $\pi_{K}$ is $\mathcal{W}_{K}:=\sigma\left(\pi_{K}\right)=\pi_{K}^{-1}(\mathcal{W})$. By (A.1) we have $\mathcal{W}_{K_{1}} \subset \mathcal{W}_{K_{2}}$ for $K_{1}, K_{2} \in \mathcal{F}(\boldsymbol{Y})$ with $K_{1} \subset K_{2}$. A stopping set (defined on $\boldsymbol{W}$ with respect to $\left.\left(\mathcal{W}_{K}\right)_{K \in \mathcal{F}(\boldsymbol{Y})}\right)$ is a mapping $T: \boldsymbol{W} \rightarrow$ $\mathcal{F}(\boldsymbol{Y})$ such that $\{\eta \in \boldsymbol{W}: T(\eta) \subset K\} \in \mathcal{W}_{K}, K \in \mathcal{F}(\boldsymbol{Y})$. The stopping $\sigma$-field associated with a stopping set $T$ is defined by $\mathcal{W}_{T}:=\left\{A \in \mathcal{W}: A \cap\{T \subset K\} \in \mathcal{W}_{K}\right.$ for all $\left.K \in \mathcal{F}(\boldsymbol{Y})\right\}$. It is easy to check that a stopping set $T$ is $W_{T}$-measurable. Indeed, if $U \subset Y$ is open then $\{T \cap U=\varnothing\} \cap\{T \subset K\}=\{T \subset K \backslash U\} \in \mathcal{W}_{K \backslash U} \subset \mathcal{W}_{K}$. 
Lemma A.1. Let $(\boldsymbol{X}, \mathcal{X})$ be a Borel space, and let $f: \boldsymbol{W} \rightarrow \boldsymbol{X}$ be a measurable function. Then $f$ is $\mathcal{W}_{K}$-measurable if and only if $f=f \circ \pi_{K}$.

Proof. Let $f$ be measurable with respect to $\mathcal{W}_{K}$. By Lemma 1.13 of [4], there exists some measurable mapping $h: \boldsymbol{W} \rightarrow \boldsymbol{X}$ with $f=h \circ \pi_{K}$. Hence, $f \circ \pi_{K}=f$ by (A.1).

For a function $T: \boldsymbol{W} \rightarrow \mathcal{F}(\boldsymbol{Y})$, we define $\pi_{T}: \boldsymbol{W} \rightarrow \boldsymbol{W}$ by $\pi_{T}(\eta):=\pi_{T(\eta)}(\eta), \eta \in \boldsymbol{W}$.

Lemma A.2. Let $T$ be a stopping set. Then the following assertions hold.

(i) $\mathcal{W}_{T}=\sigma\left(\pi_{T}\right)$ if $\pi_{T}$ is measurable.

(ii) $T(\eta)=T\left(\pi_{T}(\eta)\right)$ for all $\eta \in W$.

(iii) Let $\eta \in \boldsymbol{W}$ and $K \in \mathcal{F}(\boldsymbol{Y})$. Then $T(\eta) \subset K$ if and only if $T\left(\pi_{K}(\eta)\right) \subset K$.

(iv) Let $\eta \in \boldsymbol{W}$ and $K \in \mathcal{F}(\boldsymbol{Y})$, and assume that $T(\eta) \subset K$. Then $T(\eta)=T\left(\pi_{K}(\eta)\right)$.

Proof. To prove $\mathcal{W}_{T} \subset \sigma\left(\pi_{T}\right)$, let $A \in \mathcal{W}_{T}, K \in \mathcal{F}(\boldsymbol{Y})$, and $\eta \in \boldsymbol{W}$. By the definition of $\mathcal{W}_{T}$, the function $\mathbf{1}_{A} \mathbf{1}\{T \subset K\}$ is measurable with respect to $\mathcal{W}_{K}$. Using Lemma A.1, we obtain

$$
\mathbf{1}_{A}(\eta) \mathbf{1}\{T(\eta) \subset K\}=\mathbf{1}_{A}\left(\pi_{K}(\eta)\right) \mathbf{1}\left\{T\left(\pi_{K}(\eta)\right) \subset K\right\} .
$$

Setting $A=\boldsymbol{W}$ yields the third assertion, i.e.

$$
\mathbf{1}\{T(\eta) \subset K\}=\mathbf{1}\left\{T\left(\pi_{K}(\eta)\right) \subset K\right\} .
$$

Set $K:=T(\eta)$ and use (A.2) and (A.3) to obtain $\mathbf{1}_{A}(\eta)=\mathbf{1}_{A}\left(\pi_{T}(\eta)\right)$, which in turn implies that $A=\pi_{T}^{-1}(A) \in \pi_{T}^{-1}(\mathcal{W})=\sigma\left(\pi_{T}\right)$.

Recall that $T$ is $\mathcal{W}_{T}$-measurable. Using (A.2) with $A=T^{-1}(\{T(\eta)\}) \in \mathcal{W}_{T}$ and (A.3), we have, in the case of $T(\eta) \subset K, 1=\mathbf{1}\{T(\eta)=T(\eta)\}=\mathbf{1}\left\{T\left(\pi_{K}(\eta)\right)=T(\eta)\right\}$. Hence, the second and fourth assertions are also true.

Now we prove that $\sigma\left(\pi_{T}\right) \subset \mathcal{W}_{T}$. Let $A \in \mathcal{W}, K \in \mathcal{F}(\boldsymbol{Y})$, and $\eta \in \boldsymbol{W}$, and let $\pi_{T}$ be measurable. By (A.1), (A.3), and assertion (iv), we obtain

$$
\mathbf{1}\left\{\pi_{T}(\eta) \in A\right\} \mathbf{1}\{T(\eta) \subset K\}=\mathbf{1}\left\{\pi_{T}\left(\pi_{K}(\eta)\right) \in A\right\} \mathbf{1}\left\{T\left(\pi_{K}(\eta)\right) \subset K\right\},
$$

which implies $\mathcal{W}_{K}$-measurability of $\left\{\pi_{T} \in A\right\} \cap\{T \subset K\}$. Hence, $\left\{\pi_{T} \in A\right\} \in \mathcal{W}_{T}$, completing the proof of the first assertion.

Proposition A.1. A measurable function $T: \boldsymbol{W} \rightarrow \mathcal{F}(\boldsymbol{Y})$ is a stopping set if and only if $T(\eta)=T\left(\pi_{T}(\eta)\right)$ for all $\eta \in \boldsymbol{W}$ and the following implication holds for all $\eta, \psi \in \boldsymbol{W}$ :

$$
\psi=\pi_{T(\psi)}(\eta) \quad \Longrightarrow \quad T(\psi)=T(\eta) .
$$

Proof. Let $T$ be a stopping set, let $\psi, \eta \in W$ with $\psi=\pi_{T(\psi)}(\eta)$, and set $K:=T(\psi)$. Using Lemma A.2(iii), $\psi=\pi_{K}(\eta)$, and $T\left(\pi_{K}(\eta)\right)=T(\psi) \subset K$, we obtain $T(\eta) \subset K$. Invoking Lemma A.2(iv), we see that $T(\eta)=T\left(\pi_{K}(\eta)\right)=T(\psi)$. Note that $T(\eta)=T\left(\pi_{T}(\eta)\right)$ holds by Lemma A.2(ii).

Now assume that $T(\eta)=T\left(\pi_{T}(\eta)\right)$ and that (A.4) holds for all $\eta, \psi \in \boldsymbol{W}$. Let $\eta \in \boldsymbol{W}$ and $K \in \mathcal{F}(\boldsymbol{Y})$ with $T(\eta) \subset K$, and define $\psi:=\pi_{T}(\eta)$. We have $T(\psi)=T(\eta) \subset K$, so that, by (A.1), $\psi=\pi_{T(\psi)}(\eta)=\pi_{T(\psi)}\left(\pi_{K}(\eta)\right)$. If vice versa, there exists $\psi \in \boldsymbol{W}$ with $T(\psi) \subset K$ 
and $\psi=\pi_{T(\psi)}\left(\pi_{K}(\eta)\right)$. Then $\psi=\pi_{T(\psi)}(\eta)$ by (A.1), and (A.4) yields $T(\eta)=T(\psi) \subset K$. Hence,

$$
T(\eta) \subset K \quad \Longleftrightarrow \quad \text { there exists } \psi \in W \text { with } T(\psi) \subset K \text { and } \psi=\pi_{T(\psi)}\left(\pi_{K}(\eta)\right) \text {. }
$$

Therefore, $\mathbf{1}\{T(\eta) \subset K\}=\mathbf{1}\left\{T\left(\pi_{K}(\eta)\right) \subset K\right\}$. As $\{T \subset K\} \in \mathcal{W}$, we in fact obtain $\{T \subset K\} \in \mathcal{W}_{K}$, so that $T$ is a stopping set.

Proposition A.1 is the right tool for proving Proposition 2.1.

Proof of Proposition 2.1. Let $T^{\prime}: N^{k} \rightarrow \mathcal{F}$ be measurable such that $T:=\mathcal{E}_{T^{\prime}}^{k}$ is closed. Assume that (2.7) holds. Then $T^{\prime}$ is a stopping set with respect to $\left(\sigma\left(\pi_{K}^{\prime}\right)\right)_{K \in \mathcal{F}}$, where $\pi_{K}^{\prime}$ is defined in (2.5), i.e.

$$
\pi_{K}^{\prime}(\eta)=\{F \in \eta: F \cap K \neq \varnothing\}, \quad \eta \in N^{k}, K \in \mathcal{F} .
$$

Hence, we obtain, from Proposition A.1,

$$
T^{\prime}(\eta)=T^{\prime}\left(\pi_{T^{\prime}}^{\prime}(\eta)\right)=T^{\prime}(\eta \cap T(\eta))
$$

and the implication

$$
\psi=\eta \cap T(\psi) \quad \Longrightarrow \quad T^{\prime}(\eta)=T^{\prime}(\psi)
$$

for all $\eta, \psi \in N^{k}$. Note that $T^{\prime}(\eta)=T^{\prime}(\psi)$ implies that $T(\eta)=T(\psi)$ and (A.6) implies that $T(\eta)=T(\eta \cap T(\eta))$. By Proposition A.1, $T$ is a stopping set with respect to $\sigma\left(\pi_{K}\right)_{K \in \mathcal{F}\left(\mathcal{E}^{k}\right)}$, where $\pi_{K}$ is defined by

$$
\pi_{K}(\eta):=\eta \cap K, \quad \eta \in N^{k}, K \in \mathcal{F}\left(\mathcal{E}^{k}\right) .
$$

From $\pi_{T^{\prime}}^{\prime}=\pi_{T}$ and Lemma A.2(i), we obtain $\mathcal{N}_{T^{\prime}}^{k}=\sigma\left(\pi_{T^{\prime}}^{\prime}\right)=\sigma\left(\pi_{T}\right)=\mathcal{N}_{T}^{k}$, which proves (2.8).

The next result leads to an easy and transparent proof of Proposition 3.1.

Lemma A.3. $\operatorname{Set} \pi_{K}(\eta):=\eta \cap K, \eta \in N^{k}$, and $K \in \mathcal{F}\left(\varepsilon^{k}\right)$. Let $\eta \in N^{k}$, let $T: N^{k} \rightarrow \mathcal{F}\left(\mathcal{E}^{k}\right)$ be a stopping set, and let $f: N^{k} \times \mathcal{F}\left(\mathcal{E}^{k}\right) \rightarrow[0, \infty)$ be a measurable function. Then

$$
\begin{aligned}
& f\left(\pi_{T}(\eta), T(\eta)\right) \mathbf{1}\{\eta(T(\eta))=m\} \\
&=\frac{1}{m !} \int f\left(\left\{F_{1}, \ldots, F_{m}\right\}, T\left(\left\{F_{1}, \ldots, F_{m}\right\}\right)\right) \mathbf{1}\left\{F_{1}, \ldots, F_{m} \in T\left(\left\{F_{1}, \ldots, F_{m}\right\}\right)\right\} \\
& \times \mathbf{1}\left\{\eta_{F_{1}, \ldots, F_{m}}\left(T\left(\left\{F_{1}, \ldots, F_{m}\right\}\right)\right)=0\right\} \eta^{(m)}\left(\mathrm{d}\left(F_{1}, \ldots, F_{m}\right)\right)
\end{aligned}
$$

for $m \geq 1$ and

$$
f\left(\pi_{T}(\eta), T(\eta)\right) \mathbf{1}\{\eta(T(\eta))=0\}=f(\varnothing, T(\varnothing)) \mathbf{1}\{\eta(T(\varnothing))=0\} .
$$

Proof. We assume that $m \geq 1$. In the case in which $m=0$ the proof is similar but simpler. For any $\eta \in N^{k}$, we have

$$
\begin{aligned}
& f\left(\pi_{T}(\eta), T(\eta)\right) \mathbf{1}\{\eta(T(\eta))=m\} \\
& \quad=\frac{1}{m !} \int f\left(\left\{F_{1}, \ldots, F_{m}\right\}, T(\eta)\right) \mathbf{1}\left\{\left\{F_{1}, \ldots, F_{m}\right\}=\pi_{T}(\eta)\right\} \eta^{(m)}\left(\mathrm{d}\left(F_{1}, \ldots, F_{m}\right)\right) .
\end{aligned}
$$


From Proposition A.1 we obtain

$$
\left\{F_{1}, \ldots, F_{m}\right\}=\pi_{T}(\eta) \Longleftrightarrow\left\{F_{1}, \ldots, F_{m}\right\}=\pi_{T\left(\left\{F_{1}, \ldots, F_{m}\right\}\right)}(\eta),
$$

as well as $T(\eta)=T\left(\left\{F_{1}, \ldots, F_{m}\right\}\right)$, if the equations above are true. We conclude that

$$
\begin{aligned}
& f\left(\pi_{T}(\eta), T(\eta)\right) \mathbf{1}\{\eta(T(\eta))=m\} \\
& =\frac{1}{m !} \int f\left(\left\{F_{1}, \ldots, F_{m}\right\}, T\left(\left\{F_{1}, \ldots, F_{m}\right\}\right)\right) \\
& \times \mathbf{1}\left\{\left\{F_{1}, \ldots, F_{m}\right\}=\pi_{T\left(\left\{F_{1}, \ldots, F_{m}\right\}\right)}(\eta)\right\} \eta^{(m)}\left(\mathrm{d}\left(F_{1}, \ldots, F_{m}\right)\right) \\
& =\frac{1}{m !} \int f\left(\left\{F_{1}, \ldots, F_{m}\right\}, T\left(\left\{F_{1}, \ldots, F_{m}\right\}\right)\right) \mathbf{1}\left\{F_{1}, \ldots, F_{m} \in T\left(\left\{F_{1}, \ldots, F_{m}\right\}\right)\right\} \\
& \times \mathbf{1}\left\{\eta_{F_{1}, \ldots, F_{m}}^{!}\left(T\left(\left\{F_{1}, \ldots, F_{m}\right\}\right)\right)=0\right\} \eta^{(m)}\left(\mathrm{d}\left(F_{1}, \ldots, F_{m}\right)\right) .
\end{aligned}
$$

\section{Acknowledgements}

Several helpful comments of two anonymous referees have led to a significant improvement in the presentation of our results. We are also grateful to Jürgen Kampf for many useful remarks on properties of finitely generated hyperplane tessellations; see Remark 6.4.

\section{References}

[1] Baumstark, V. and Last, G. (2007). Some distributional results for Poisson-Voronoi tessellations. Adv. Appl. Prob. 39, 16-40.

[2] CowAn, R. (2006). A more comprehensive complementary theorem for the analysis of Poisson point processes. Adv. Appl. Prob. 38, 581-601.

[3] Cowan, R., Quine, M. And Zuyev, S. (2003). Decomposition of gamma-distributed domains constructed from Poisson point processes. Adv. Appl. Prob. 35, 56-69.

[4] Kallenberg, O. (2002). Foundations of Modern Probability, 2nd edn. Springer, New York.

[5] LAst, G. AND Thorisson, H. (2009). Invariant transports of stationary random measures and mass-stationarity. Ann. Prob. 37, 790-813.

[6] Matheron, G. (1975). Random Sets and Integral Geometry. John Wiley, New York.

[7] Mecke, J. (1967). Stationäre zufällige Maße auf lokalkompakten Abelschen Gruppen. Z. Wahrscheinlichkeitsth. 9, 36-58.

[8] Miles, R.E. (1971). Poisson flats in Euclidean spaces. II. Homogeneous Poisson flats and complementary theorem. Adv. Appl. Prob. 3, 1-43.

[9] Miles, R. E. (1974). A synopsis of 'Poisson flats in Euclidean spaces'. In Stochastic Geometry, eds E. F. Harding and D. G. Kendall, John Wiley, New York, pp. 202-227.

[10] Miles, R. E. And Maillardet, R. J. (1982). The basic structure of Voronoi and generalized Voronoi polygons. In Essays in Statistical Science (J. Appl. Prob. Spec. Vol. 19A), eds J. Gani and E. J. Hannan, Applied Probability Trust, Sheffield, pp. 97-111.

[11] MøLler, J. (1994). Lectures on Random Voronoi Tessellations (Lecture Notes Statist. 87). Springer, New York.

[12] Møller, J. ANd ZuYev, S. (1996). Gamma-type results and other related properties of Poisson processes. Adv. Appl. Prob. 28, 662-673.

[13] Muche, L. (2005). The Poisson-Voronoi tessellation: relationships for edges. Adv. Appl. Prob. 37, $279-296$.

[14] SchneIder, R. AND WeIL, W. (2008). Stochastic and Integral Geometry. Springer, Berlin.

[15] Stoyan, D., Kendall, W. S. And Mecke, J. (1995). Stochastic Geometry and Its Applications, 2nd edn. John Wiley, Chichester.

[16] Z̈̈HLE, M. (1982). Random processes of Hausdorff rectifiable closed sets. Math. Nachr. 108, 49-72.

[17] ZuYev, S. (1999). Stopping sets: gamma-type results and hitting properties. Adv. Appl. Prob. 31, 355-366. 